\title{
Nonlinear dynamic characteristics of FGCNTs reinforced microbeam with piezoelectric layer based on unifying stress-strain gradient framework
}

\author{
W.D. Yang, C.Q. Fang and X. Wang ${ }^{*}$ \\ School of Naval Architecture, Ocean and Civil Engineering \\ (State Key Laboratory of Ocean Engineering) \\ Shanghai Jiao Tong University, Shanghai 200240, P. R. China
}

\begin{abstract}
A size-dependent model of functionally graded carbon nanotubes (FGCNTs) reinforced microbeam with piezoelectric layer is presented based on unifying nonlocal stress and strain gradient framework. Nonlinear dynamic characteristics of the microbeam arise from electrostatic, piezoelectric actuation and thermal loading, with consideration of quantum and thermal fluctuations induced Casimir force. The nonlinearly dynamic frequency and pull-in instability of FGCNTs reinforced microbeam with damping effect are investigated by perturbation technique and verified by numerical method, where the coupling effects of nonlocal stress gradient and strain gradient parameters on fundamental frequency are described. The results show that the frequency of piezoelectric laminated microbeam-damping system declines with the growth of nonlocal stress gradient parameter while increases with the increment of strain gradient parameter. The pull-in voltage of micro- piezoelectric laminated beam can be tuned by excitation voltage exerted on piezoelectric layer, and the frequency and pull-in voltage decrease with the increase of the excitation voltage exerted on piezoelectric layer. In addition, dissipative effects originating from viscous and structural damping are evaluated. It is found that the pull-in voltage of micro-structures with damping system is higher than that of undamped system.
\end{abstract}

Key words: Nonlinearly dynamic pull-in, Piezoelectric layer, Nonlocal stress-strain gradients, Functionally graded carbon nanotubes composite, Thermal Casimir force

\footnotetext{
${ }^{*}$ Corresponding author at: School of Naval Architecture, Ocean and Civil Engineering ,Shanghai Jiaotong University, Shanghai 200240, P. R. China.

Tel./fax: 086-021-54745367/086-021-54745367

E-mail address: xwang@sjtu.edu.cn (X.Wang).
} 


\section{Introduction}

Micro-/nano-electromechanical systems (M/NEMS) have attracted considerable attention from academic and industrial communities due to their advantages of small size, low weight, rapid response, relatively intensive and low cost [1]. These characteristics collectively render M/NEM-based devices suitable for a wide range of promising applications in micro-/nano-scale sensors, actuators, switches, and resonators [2-6].

One critical phenomenon of electrostatically actuated devices is pull-in instability, where the balance between Coulomb force and elastic restoring force is lost and accordingly the system collapses onto the substrate [7]. To avoid such failure, the laminated structures with piezoelectric layers are proposed to control or tune the pull-in voltage by piezoelectric effect [8-13]. Because piezoelectric materials have a significant strength of high force transmission with low driving voltage, the hybrid electrostatic and piezoelectric microbeam devices are proved to possess the greater efficiency, reduce energy consumption and effectively control pull-in voltage by piezoelectric layer actuation.

However, dispersion forces exhibiting dominant role in micro-structures were not considered in above studies of electrostatically actuated microbeam with piezoelectric layer(s). The schemes of different types of dispersion forces [14, 15] are shown in Fig.

1: (a) van der Waals force interacting between neutral particles like atoms and molecules; (b) Casimir-Polder force dominating between neutral particle and macroscopic solid; (c) quantum Casimir force between two macroscopic bodies at zero temperature; (d) complete description of Casimir force with quantum and thermal fluctuations of electromagnetic field at finite temperature. Note that Casimir force originates from two paths of quantum and thermal fluctuations of electromagnetic waves $[16,17]$. Although there exist a great deal of literature on pull-in instability considering quantum Casimir force at zero temperature $[3,5,6$, $18,19]$, the assumption of zero temperature is not accurate enough, and thermal Casimir force is few reported to exploit the electrostatic devices. Therefore, thermal 
fluctuation effects should be considered on electromechanical behaviors of microstructure at finite temperature.

Continuing miniaturization of devices calls conventional continuum theories into question due to their inability to capture size-dependent characteristics of micro-/nano-scale materials. In the past decade, several modified elasticity theories were proposed to study mechanical properties of microstructure with small scale effect. These modified theories mainly incorporate two types: (i) stress gradient model based on Eringen's nonlocal elasticity [20, 21], which considers long-range interactions between atoms of solids; (ii) strain gradient model including (modified) couple stress theory [22, 23] and strain gradient theory [24], which postulate mechanical response of material at a point relies not only on the local strain, but also on higher-order strain gradients.

There have been plentiful applications of nonlocal stress gradient model and strain gradient model to investigate the pull-in instability of microstructures. The results show that the stress gradient model only reflects the softening effect of structural stiffness, which are against the hardening effect observed from experiment as well as strain gradient model $[3,5,25,26]$. Lim et al. proposed one higher-order nonlocal stress gradient and strain gradient model to investigate the wave propagation of carbon nanotubes (CNTs) based on nanobeam model [27]. Li et al. utilized nonlocal stress-strain gradient model to evaluate the buckling and flexural wave propagation of nanobeam $[28,29]$. It is seen from literature that the size-dependent mechanical response of micro-/nano-scale structures depends on coupling influences of nonlocal stress gradient and strain gradient parameters.

Furthermore, functionally graded materials (FGMs) have become a promising branch of composite materials due to their properties varying spatially in terms of non-uniform distribution of the reinforcing phase $[30,31]$. In particular, CNTs reinforced composites (CNTsRC) have been widely utilized in the field of nano-composite structures because of super high strength and stiffness ratio of CNTs [32,33]. There have been numerous papers on the nonlinear bending [34], free vibration [35], buckling [36] problems of functionally graded CNTs (FGCNTs) 
reinforced composites. Recently, Kiani et al. investigated the nonlinear dynamics and thermal analysis of FGCNTs reinforced composite face sheets, such as snap-through [37], free vibration [38], low velocity impact [39] and thermal postbuckling characteristics [40]. They utilized temperature-dependent sandwich beam model to study the influences of CNTs distribution pattern, volume faction and thermal environment on fundamental frequency and dynamic stability of sandwich beam.

This work aims to investigate dynamic behaviors and pull-in instability of FGCNTs reinforced microbeam with piezoelectric layer based on unifying nonlocal stress-strain gradient model, where the microbeam is subjected to electrostatic and piezoelectric actuation incorporating quantum and thermal Casimir effect. The coupling influences of nonlocal stress and strain gradient parameters on dynamic behaviors of microbeam with piezoelectric layer are also discussed. The main results and new conclusions obtained may be useful references for application and design of micro-/nano-electromechanical systems with piezoelectric layer.

\section{Theoretical model and governing equation}

The FGCNTs reinforced microbeam with piezoelectric layer under electrostatic and dispersion forces is depicted in Fig. 2(a, b). The FGCNTs microbeam is regarded as the long and thin beam with the length $L$, width $b$, where the thicknesses of elastic microbeam and piezoelectric layer are $h_{e}$ and $h_{p}$, respectively. The initial gap between two electrodes is $g$. The coordinate system is located to the neutral axis at middle-left end of microbeam, where $x$ and $z$ are the horizontal and perpendicular directions.

\subsection{Material properties of FGCNTs composites with piezoelectric layer}

Considering that the material property of CNTsRC microbeam depends on environment temperature, the effective Young's modulus of CNTsRC is determined by the single material parameter $E_{\text {eff }}^{e}$, so that the effective material properties of CNTsRC with temperature-dependency are expressed in terms of the extensive rule of 
mixture as [35]

$$
\begin{aligned}
& E_{\text {eff }}^{e}(z, T)=\eta_{1} V_{c n t}(z) E_{c n t}(T)+V_{m}(z) E_{m}(T) \\
& \alpha_{e f f}^{e}(z, T)=V_{c n t}(z) \alpha_{c n t}(T)+V_{m}(z) \alpha_{m}(T) \\
& \rho_{e f f}^{e}(z, T)=V_{c n t}(z) \rho_{c n t}(T)+V_{m}(z) \rho_{m}(T)
\end{aligned}
$$

where $E_{e f f}^{e}, E_{c n t}$ and $E_{h}$ are temperature-dependent effective modulus of CNTsRC, Young's modulus of CNTs and matrix; $\alpha_{\text {eff }}^{e}$ and $\rho_{\text {eff }}^{e}$ are effective thermal expansion coefficient and mass density of CNTsRC; $\alpha_{c n t}$ and $\alpha_{m}$ are thermal expansion coefficients of CNTs and matrix. Additionally, the material properties of the piezoelectric layer as Young's modulus $E^{p}$, thermal expansion coefficient $\alpha^{p}$ and mass density $\rho^{p}$ are considered as independent of temperature.

Here, CNTs reinforcement is considered to be uniformly distributed (UD-CNTsRC) or functionally graded in thickness direction of microbeam, such as O-type (FGO-CNTsRC), X-type (FGX-CNTsRC), A-type (FGA-CNTsRC) and V-type (FGV-CNTsRC) illustrated as in Fig.2(b) [41]. The geometrical distribution of CNTs is, respectively, considered as

$$
\begin{array}{ll}
V_{c n t}(z)=V_{c n t}^{*}, & (\mathrm{UD}-\mathrm{CNTsRC}) \\
V_{c n t}(z)=2\left(1-2|z| / h_{e}\right) V_{c n t}^{*}, & (\mathrm{FGO}-\mathrm{CNTsRC}) \\
V_{c n t}(z)=2\left(2|z| / h_{e}\right) V_{c n t}^{*}, & (\mathrm{FGX}-\mathrm{CNTsRC}) \\
V_{c n t}(z)=\left(1-2 z / h_{e}\right) V_{c n t}^{*}, & (\mathrm{FGA}-\mathrm{CNTsRC}) \\
V_{c n t}(z)=\left(1+2 z / h_{e}\right) V_{c n t}^{*}, & (\mathrm{FGV}-\mathrm{CNTsRC})
\end{array}
$$

where

$$
V_{c n t}^{*}=\frac{m_{c n t}}{m_{c n t}+\rho_{c n t} / \rho_{m}-\left(\rho_{c n t} / \rho_{m}\right) m_{c n t}}
$$

and $m_{c n t}$ is the mass fraction of CNTs; $\rho_{c n t}$ and $\rho_{m}$ are mass densities of CNTs and matrix, respectively. 
For the thin-long microbeam, the Young's modulus of CNTsRC only depends on $E_{c n t}^{11}$ and $\eta_{1}$, so that according to the rule of mixture [35]错误! 未定义书签。, the general effective material properties of CNTsRC are expressed by

$$
E_{c n t}(T)=\eta_{1} V_{c n t} E_{c n t}^{11}(T)+V_{m} E_{m}(T)
$$

where $E_{c n t}^{11}$ is the longitudinal Young's modulus of CNTs, $E_{m}$ is the Young's modulus of the matrix, and $\eta_{1}$ is the CNTs efficient parameter for the scale-dependent material properties, determined by MD simulation. $V_{c n t}$ and $V_{m}$ are the volume fractions of CNTs and matrix, satisfying $V_{c n t}+V_{m}=1$.

Note that the physical neutral axis of the FGCNTs reinforced microbeam with piezoelectric layer is no longer located at the geometric reference axis. According to the sum of bending moments are zero at the physical neutral axis [42], satisfying

$$
\int_{-\frac{1}{2} h_{e}}^{\frac{1}{2} h_{e}} E_{e f f}^{e}\left(z-z_{n}\right) d z+\int_{-\frac{1}{2} h_{e}-h_{p}}^{-\frac{1}{2} h_{e}} E^{p}\left(z-z_{n}\right) d z=0
$$

From Eq. (5), the physical neutral axis of bilayer structure is written as

$$
Z_{n}=\frac{\int_{-\frac{1}{2} h_{e}}^{\frac{1}{2} h_{e}} E_{\text {eff }}^{e} z d z+\int_{-\frac{1}{2} h_{e}-h_{p}}^{-\frac{1}{2} h_{e}} E^{p} z d z}{\int_{-\frac{1}{2} h_{e}}^{\frac{1}{2} h_{e}} E_{\text {eff }}^{e} d z+\int_{-\frac{1}{2} h_{e}-h_{p}}^{-\frac{1}{2} h_{e}} E^{p} d z}
$$

\subsection{Nonlocal stress-strain gradient elasticity}

According to the nonlocal stress-strain gradient elasticity theory, the influence of higher-order strain gradients are considered except for nonlocal effects of the classical strain field. The extended internal energy density potential is expressed as [27]

$$
\begin{aligned}
U_{0}\left(\varepsilon_{i j}, \varepsilon_{i j}^{\prime}, \alpha_{0} ; \varepsilon_{i j, m}, \varepsilon_{i j, m}^{\prime}, \alpha_{1}\right)= & \frac{1}{2} \varepsilon_{i j} C_{i j k l} \int_{V} \alpha_{0}\left(\left|x-x^{\prime}\right|, e_{0} a\right) \varepsilon^{\prime}{ }_{k l} d V \\
& +\frac{1}{2} \tilde{l}^{2} \varepsilon_{i j, m} C_{i j k l} \int_{V} \alpha_{1}\left(\left|x-x^{\prime}\right|, e_{1} a\right) \varepsilon^{\prime}{ }_{k l, m} d V
\end{aligned}
$$

where $\varepsilon_{i j, m}$ and $\varepsilon_{i j, m}^{\prime}$ are higher-order counterparts of strain tensors at point $x$ and 
$X^{\prime} ; \alpha_{1}$ is another attenuation kernel function representing the nonlocal effect of the first-order strain gradient field; $\tilde{l}$ and $e_{1}$ are the material length scale originating from higher-order statin gradient field and related material constant, respectively.

The higher-order nonlocal stress-strain gradient elasticity can be further simplified by assuming $e_{1}=e_{0}$ and keeping related terms of order $O\left(\nabla^{2}\right)$, the reduced model incorporating unified nonlocal stress-strain gradients is expressed as [27]

$$
\left[1-\left(e_{0} a\right)^{2} \nabla^{2}\right] \sigma_{x x}=E_{e f f}\left(1-l_{0}^{2} \nabla^{2}\right) \varepsilon_{x x}
$$

where $E_{\text {eff }}$ is effective Young's modulus of bulk. Note that the nonlocal stress gradient constitutive relation can be gained by setting strain gradient material length scale $l_{0}=0$, and the single strain gradient constitutive relation can be obtained by setting nonlocal stress gradient material constant $e_{0}=0$.

\subsection{Quantum and thermal dispersion forces}

FGCNTs reinforced microbeam with piezoelectric layer is subjected to electrostatic load incorporating first-order correction fringing field, as shown in Fig. 2(a). The electrostatic force per unit length exerted on the microbeam is defined by [5]

$$
F_{e}=\frac{\varepsilon_{0} \bar{V}_{e}^{2} b}{2[g-w(x)]^{2}}\left[1+\frac{\gamma_{0}}{b}(g-w(x))\right]
$$

where $\varepsilon_{0}=8.854 \times 10^{-12} C^{2} N^{-1} m^{-2}$ is the permittivity of vacuum, $\bar{V}_{e}$ is the applied electrostatic voltage, $\gamma_{0}$ is the linear correction of fringing field and $g$ is the initial gap between two electrodes.

The idealized dispersion forces without thermal effects such as van der Waals force and quantum Casimir force are derived by vacuum quantum fluctuation of the electromagnetic field modes of a cavity within two perfect flat bodies, and the idealized expressions of the dispersion forces at zero temperature are, respectively [3] 


$$
\begin{aligned}
& F_{v d W}=\frac{\bar{A} b}{6 \pi[g-w(x)]^{3}} \\
& F_{C}=\frac{\pi^{2} \hbar c_{0} b}{240[g-w(x)]^{4}}
\end{aligned}
$$

where $\bar{A}$ is the Hamaker constant, $\hbar=1.055 \times 10^{-34} \mathrm{Js}$ is the reduced Plank's constant and $c_{0}=3.0 \times 10^{8} \mathrm{~m} \cdot \mathrm{s}^{-1}$ is the speed of light.

Based on the Lifshitz's theory [43], thermal Casimir force not only depends on gap distance, but also are dependent on the permittivity of materials in the complex way. When the thermal fluctuation effect of electromagnetic field at the finite environment temperature is considered, Drude model and plasma model are utilized to predict thermal Casimir force $[19,44]$, as follows

$$
F_{C}^{(T)}=\frac{\pi^{2} \hbar c b}{240[g-w(x)]^{4}}\left\{1+\frac{1}{3}\left[\frac{2(g-w(x)) k_{B} T}{\hbar c}\right]^{4}\right\}+\frac{k_{B} T \zeta(3)}{4 \pi^{2}[g-w(x)]^{3}}
$$

where $k_{B}$ is Boltzmann's constant, $T$ is environment temperature and $\zeta(3)=1.202$ is the Riemann zeta function. When $T \rightarrow 0$, Eq.(12) is reduced to quantum Casimir force $F_{C}$, Eq. (11) without considering thermal fluctuation effects.

\subsection{Governing equation of microbeam with piezoelectric layer}

Based on the Euler-Bernoulli beam theory, the axial displacement $u_{x}$ and the transverse displacement of any points at microbeam $u_{z}$ are expressed as

$$
u_{x}(x, z, t)=u(x, t)-z \frac{\partial w(x, t)}{\partial x}, \quad u_{z}(x, z, t)=w(x, t)
$$

where $u(x, t)$ and $w(x, t)$ are the axial and transverse displacements of the mid-plane at time $t$. The strain-displacement relation in terms of von Kármán-type geometrical nonlinearity is given by

$$
\varepsilon_{x x}=\frac{\partial u_{x}}{\partial x}+\frac{1}{2}\left(\frac{\partial u_{z}}{\partial x}\right)^{2}=\frac{\partial u}{\partial x}-z \frac{\partial^{2} w}{\partial x^{2}}+\frac{1}{2}\left(\frac{\partial w}{\partial x}\right)^{2}
$$

Based on Hamilton's principle, the motion equations of Euler-Bernoulli beam 
with damping systems are expressed as [45]

$$
\begin{gathered}
\frac{\partial N_{x}}{\partial x}+F_{x}=I_{1} \frac{\partial^{2} u}{\partial t^{2}} \\
\frac{\partial^{2} M_{x}}{\partial x^{2}}+\frac{\partial}{\partial x}\left(N_{x} \frac{\partial w}{\partial x}\right)+F_{z}-C_{v d} \frac{\partial w}{\partial t}-C_{s d} \frac{\partial^{2} w}{\partial t \partial x}=I_{1} \frac{\partial^{2} w}{\partial t^{2}}
\end{gathered}
$$

where $N_{x}, M_{x}$ are, respectively, the axial force and bending moment, as follows

$$
\begin{aligned}
& N_{x}=\int_{-\frac{1}{2} h_{e}-z_{n}}^{\frac{1}{2} h_{e}-z_{n}} \sigma_{x x}^{e} b d z+\int_{-\frac{1}{2} h_{e}-z_{n}-h_{p}}^{-\frac{1}{2} h_{e}-z_{n}} \sigma_{x x}^{p} b d z \\
& M_{x}=\int_{-\frac{1}{2} h_{e}-z_{n}}^{\frac{1}{2} h_{e}-z_{n}} \sigma_{x x}^{e} b z d z+\int_{-\frac{1}{2} h_{e}-z_{n}-h_{p}}^{-\frac{1}{2} h_{e}-z_{n}} \sigma_{x x}^{p} b z d z
\end{aligned}
$$

, and dissipative effects of dynamic system are represented by the structural and viscous damping with coefficients $C_{s d}$ and $C_{v d}$, respectively. $F_{x}, F_{z}$ are applied axial and transverse forces, and $I_{1}$ is the inertia of microbeam with piezoelectric layer given by

$$
I_{1}=\int_{-\frac{1}{2} h_{e}}^{\frac{1}{2} h_{e}} \rho_{e f f}^{e}(z, T) b d z+\int_{-\frac{1}{2} h_{e}-h_{p}}^{-\frac{1}{2} h_{e}} \rho^{p} b d z
$$

Considering that the microbeam with piezoelectric layer is immersed in thermal environment of temperature change and utilizing Eqs.(8) and (14), the constitutive relations for nonlocal stress-strain gradients are, respectively, expressed by

$$
\begin{aligned}
& {\left[1-\left(e_{0} a\right)^{2} \nabla^{2}\right] \sigma_{x x}^{e(n l)}=E_{\text {eff }}^{e}(z, T)\left(1-l_{0}^{2} \nabla^{2}\right)\left[\frac{\partial u}{\partial x}-z \frac{\partial^{2} w}{\partial x^{2}}+\frac{1}{2}\left(\frac{\partial w}{\partial x}\right)^{2}-\alpha_{e f f}^{e}(z, T) \Delta T\right]} \\
& {\left[1-\left(e_{0} a\right)^{2} \nabla^{2}\right] \sigma_{x x}^{p(n l)}=E^{p}(z, T)\left(1-l_{0}^{2} \nabla^{2}\right)\left[\frac{\partial u}{\partial x}-z \frac{\partial^{2} w}{\partial x^{2}}+\frac{1}{2}\left(\frac{\partial w}{\partial x}\right)^{2}-\alpha^{p} \Delta T-d_{31} E_{z}\right]}
\end{aligned}
$$

where $\sigma_{x x}^{e(n l)}$ and $\sigma_{x x}^{p(n l)}$ denote nonlocal axial stresses of microbeam and piezoelectric layer; $e_{0}$ and $a$ are the material constant and the internal characteristic length [46]. $d_{31}$ is the piezoelectric constant and $E_{z}$ is the electric field intensity determined by electric potential $V_{p}$ exerting on piezoelectric layer as $E_{z}=-V_{p} / h_{p} \quad$ [47]. 
Integrating Eqs.(20a) and (20b), the nonlocal axial internal force $N_{x}^{(n l)}$ and bending moment $M_{x}^{(n l)}$ are given by

$$
\begin{aligned}
& {\left[1-\left(e_{0} a\right)^{2} \nabla^{2}\right] N_{x}^{(n l)}=\left(1-l_{0}^{2} \nabla^{2}\right)\left[\tilde{A} \frac{\partial u}{\partial x}+\frac{1}{2} \tilde{A}\left(\frac{\partial w}{\partial x}\right)^{2}-\tilde{B} \frac{\partial^{2} w}{\partial x^{2}}\right]-N^{T}-N^{p}} \\
& {\left[1-\left(e_{0} a\right)^{2} \nabla^{2}\right] M_{x}^{(n l)}=\left(1-l_{0}^{2} \nabla^{2}\right)\left[\tilde{B} \frac{\partial u}{\partial x}+\frac{1}{2} \tilde{B}\left(\frac{\partial w}{\partial x}\right)^{2}-\tilde{D} \frac{\partial^{2} w}{\partial x^{2}}\right]-M^{T}-M^{p}}
\end{aligned}
$$

where $\{\tilde{A}, \tilde{B}, \tilde{D}\}=\int_{-\frac{1}{2} h_{e}-Z_{n}-h_{p}}^{\frac{1}{2} h_{e}-Z_{n}} E_{\text {eff }} b\left\{1, z, z^{2}\right\} d z$ are, respectively, the stretching stiffness and bending stiffness, $N^{p}$ and $M^{p}$ are the axial internal force and bending moment induced by the electric effect from piezoelectric layer, as follows

$$
\left\{N^{p}, M^{p}\right\}=\int_{-\frac{1}{2} h_{e}-Z_{n}-h_{p}}^{-\frac{1}{2} h_{e}-Z_{n}} E^{p} b d_{31} E_{z}\{1, z\} d z
$$

and $N^{T}, M^{T}$ are the axial internal force and bending moment induced by the temperature change from microbeam and piezoelectric layer, as follows:

$$
\begin{aligned}
& N^{T}=\int_{-\frac{1}{2} h_{e}-Z_{n}}^{\frac{1}{2} h_{e}-Z_{n}} E_{\text {eff }}^{e}(z, T) \alpha_{\text {eff }}^{e}(z, T) \Delta T b d z+\int_{-\frac{1}{2} h_{e}-Z_{n}-h_{p}}^{-\frac{1}{2} h_{e}-Z_{n}} E^{p} \alpha^{p} \Delta T b d z \\
& M^{T}=\int_{-\frac{1}{2} h_{e}-Z_{n}}^{\frac{1}{2} h_{e}-Z_{n}} E_{\text {eff }}^{e}(z, T) \alpha_{\text {eff }}^{e}(z, T) \Delta T z b d z+\int_{-\frac{1}{2} h_{e}-Z_{n}-h_{p}}^{-\frac{1}{2} h_{e}-Z_{n}} E^{p} \alpha^{p} \Delta T z b d z
\end{aligned}
$$

Substituting Eq.(21a) into Eq.(15), and neglecting the applied axial force and the axial inertial and rotational inertial terms, yield

$$
\tilde{A}\left[u+\int_{0}^{x} \frac{1}{2}\left(\frac{\partial w}{\partial x}\right)^{2} d x\right]=x C_{1}(t)+C_{2}(t)
$$

Utilizing the clamped ends conditions of micro-beam ( $u=0$ at $x=0$ and $L)$ and integrating Eq.(24) result in

$$
\left.u\right|_{0} ^{L}=\int_{0}^{L}\left[\frac{1}{\tilde{A}}\left(N_{x}^{(n l)}+N^{T}+N^{p}\right)-\frac{1}{2}\left(\frac{\partial w}{\partial x}\right)^{2}\right] d x=0
$$

Because the axial internal stress is a certain constant, Eq.(25) gives 


$$
N_{x}^{(n l)}=N_{x 0}^{(n l)}=\frac{\tilde{A}}{L} \int_{0}^{L}\left[\frac{1}{2}\left(\frac{\partial w}{\partial x}\right)^{2}\right]-N^{T}-N^{p}
$$

Substituting Eq.(16) and Eq.(26) into Eq.(21b) to eliminate $\partial^{2} M_{x}^{(n l)} / \partial x^{2}$, the nonlocal stress-strain gradient bending moment $M_{x}^{(n l)}$ is written as

$$
\begin{aligned}
M_{x}^{(n l)}= & \left(e_{0} a\right)^{2}\left[I_{1} \frac{\partial^{2} w}{\partial t^{2}}-\frac{\partial}{\partial x}\left(N_{x}^{(n l)} \frac{\partial w}{\partial x}\right)-F_{z}+C_{v d} \frac{\partial w}{\partial t}\right. \\
& \left.+C_{s d} \frac{\partial^{2} w}{\partial t \partial x}\right]-\tilde{D}\left(1-l_{0}^{2} \frac{\partial^{2}}{\partial x^{2}}\right) \frac{\partial^{2} w}{\partial x^{2}}-M^{T}-M^{p} \\
= & \left(e_{0} a\right)^{2}\left\{I_{1} \frac{\partial^{2} w}{\partial t^{2}}-\left[\frac{\tilde{A}}{L} \int_{0}^{L} \frac{1}{2}\left(\frac{\partial w}{\partial x}\right)^{2} d x-N^{T}-N^{p}\right] \frac{\partial^{2} w}{\partial x^{2}}-F_{z}\right. \\
& \left.+C_{v d} \frac{\partial w}{\partial t}+C_{s d} \frac{\partial^{2} w}{\partial t \partial x}\right\}-\tilde{D}\left(1-l_{0}^{2} \frac{\partial^{2}}{\partial x^{2}}\right) \frac{\partial^{2} w}{\partial x^{2}}-M^{T}-M^{p}
\end{aligned}
$$

Then substituting Eq.(26) and Eq.(27) into Eq.(16) generates the nonlinearly governing equation of microbeam with piezoelectric layer, expressed as

$$
\begin{gathered}
\left\{\tilde{D}+\left(e_{0} a\right)^{2}\left[\frac{\tilde{A}}{L} \int_{0}^{L} \frac{1}{2}\left(\frac{\partial w}{\partial x}\right)^{2} d x-N^{T}-N^{p}\right]\right\} \frac{\partial^{4} w}{\partial x^{4}}-\tilde{D} l_{0}^{2} \frac{\partial^{6} w}{\partial x^{6}}+I_{1} \frac{\partial^{2} w}{\partial t^{2}} \\
-\left(e_{0} a\right)^{2} I_{1} \frac{\partial^{4} w}{\partial t^{2} \partial x^{2}}-\left[\frac{\tilde{A}}{L} \int_{0}^{L} \frac{1}{2}\left(\frac{\partial w}{\partial x}\right)^{2} d x-N^{T}-N^{p}\right] \frac{\partial^{2} w}{\partial x^{2}} \\
-\left[1-\left(e_{0} a\right)^{2} \frac{\partial^{2}}{\partial x^{2}}\right]\left(F_{z}-C_{v d} \frac{\partial w}{\partial t}-C_{s d} \frac{\partial^{2} w}{\partial t \partial x}\right)=0
\end{gathered}
$$

where the nonlocal stress gradient governing equation can be gained by $\operatorname{setting} l_{0}=0$ and the pure strain gradient governing equation can be given by setting $e_{0}=0$. Then substituting transversely applied forces $F_{z}=F_{e}+(1-n) F_{v d W}+n F_{C}^{(T)}$ into Eq. (28) leads to the normalized form of nonlinearly governing equation, as follows 


$$
\begin{aligned}
& K_{3} \frac{\partial^{4} W}{\partial \xi^{4}}+\mu\left[K_{1} \int_{0}^{1}\left(\frac{\partial W}{\partial \xi}\right)^{2} d \xi-K_{2}\left(N^{T}+N^{p}\right)\right] \frac{\partial^{4} W}{\partial \xi^{4}}-\eta K_{3} \frac{\partial^{6} W}{\partial \xi^{6}}+K_{4} \frac{\partial^{2} W}{\partial \tau^{2}}-\mu K_{4} \frac{\partial^{4} W}{\partial \xi^{2} \partial \tau^{2}} \\
& -\left[K_{1} \int_{0}^{1}\left(\frac{\partial W}{\partial \xi}\right)^{2} d \xi-K_{2}\left(N^{T}+N^{p}\right)\right] \frac{\partial^{2} W}{\partial \xi^{2}}+C_{s d} \frac{\partial^{2} W}{\partial \tau \partial \xi}+C_{v d} \frac{\partial W}{\partial \tau}-\mu \text { 缷d } \frac{\partial^{4} W}{\partial \tau \partial \xi^{3}}-\mu C_{v d} \frac{\partial^{3} W}{\partial \tau \partial \xi^{2}} \\
& -\left(1-\mu \frac{\partial^{2}}{\partial \xi^{2}}\right)\left[\frac{V_{e}^{2} \gamma}{1-W}+\frac{V_{e}^{2}}{(1-W)^{2}}+\frac{n \beta_{2}+(1-n) \alpha_{3}}{(1-W)^{3}}+\frac{n \alpha_{4}}{(1-W)^{4}}+n \beta_{1}\right]=0
\end{aligned}
$$

where the normalized variables are introduced as follows

$$
\begin{gathered}
\xi=\frac{x}{L}, W=\frac{W}{g}, \tau=t \sqrt{\frac{D_{m}}{I_{m} L^{4}}} \\
K_{1}=\frac{\tilde{A} g^{2}}{2 D_{m}}, K_{2}=\frac{L^{2}}{D_{m}}, K_{3}=\frac{\tilde{D}}{D_{m}}, K_{4}=\frac{I_{1}}{I_{m}}, \mu=\left(\frac{e_{0} a}{L}\right)^{2}, \eta=\left(\frac{I_{0}}{L}\right)^{2}, \\
\hat{C}_{s d}=\frac{L}{\sqrt{D_{m} I_{m}}} C_{s d}, \hat{C}_{v d}=\frac{L^{2}}{\sqrt{D_{m} I_{m}}} C_{v d}, V_{e}=\bar{V}_{e} \sqrt{\frac{\varepsilon_{0} b L^{4}}{2 g^{3} D_{m}}}, \gamma_{0}=\frac{\gamma g}{b}, \\
\alpha_{3}=\frac{\bar{A} b L^{4}}{6 \pi g^{4} D_{m}}, \alpha_{4}=\frac{\pi^{2} \hbar c_{0} b L^{4}}{240 g^{5} D_{m}}, \beta_{1}=\frac{\pi^{2} \hbar c_{0} b L^{4}}{45 g D_{m}}\left(\frac{k_{B} T}{\hbar c_{0}}\right)^{4}, \beta_{2}=\frac{k_{B} T b L^{4} \zeta(3)}{4 \pi^{2} g^{4} D_{m}}
\end{gathered}
$$

where $D_{m}$ and $I_{m}$ represent bending stiffness and inertia of microbeam without CNTs. Consider normalized clamped-clamped boundary conditions as

$$
\left.W(\xi, \tau)\right|_{\xi=0}=\left.\frac{\partial W(\xi, \tau)}{\partial \xi}\right|_{\xi=0}=0,\left.W(\xi, \tau)\right|_{\xi=1}=\left.\frac{\partial W(\xi, \tau)}{\partial \xi}\right|_{\xi=1}=0
$$

To evaluate the dynamic characteristics of microbeam, using the Eigen function expansion the solution of Eq. (29) is considered as

$$
W(\xi, \tau)=\sum_{i=1}^{N} q_{i}(\tau) \phi_{i}(\xi)
$$

where $N$ represents the number of modes and $\phi_{i}(\xi)$ is the $i$-th mode shape of microbeam. Substituting the displacement function (31b) into Eq. (29), using Taylor's series expansion for electrostatic and dispersion forces and using Galerkin method [48], the following equation are obtained: 


$$
\begin{aligned}
& \sum_{i=1}^{N} \Theta_{0} \ddot{q}_{i}(\tau)+\sum_{i=1}^{N} \Theta_{6} \dot{q}_{i}(\tau)+\sum_{i=1}^{N} \sum_{j=1}^{N} \sum_{k=1}^{N} \sum_{m=1}^{N} \Theta_{5} q_{i}(\tau) q_{j}(\tau) q_{k}(\tau) q_{m}(\tau) \\
& +\sum_{i=1}^{N} \sum_{j=1}^{N} \sum_{k=1}^{N} \Theta_{4} q_{i}(\tau) q_{j}(\tau) q_{k}(\tau)+\sum_{i=1}^{N} \sum_{j=1}^{N} \Theta_{3} q_{i}(\tau) q_{j}(\tau)+\sum_{i=1}^{N} \Theta_{2} q_{i}(\tau)+\Theta_{1}=0
\end{aligned}
$$

where $\Theta_{m}, m \in[0,6]$ are given in Appendix A.

\section{Solution method}

When a step voltage is applied on the FGCNTs reinforced microbeam with piezoelectric layer, the energy of system is stored as kinetic and potential energy, where, the system without applied voltage is at rest and has no stored energy. Over time, the stored energy before related to the equilibrium position is dissipated by damping effects (i.e. structural and viscous damping). According to energy balance of the system at any instant of time, one obtains [49]

$$
E_{\text {system }}=E_{\text {kinetic }}+E_{\text {potential }}+E_{\text {dissipated }}
$$

where $E_{\text {system }}$ is the total energy of system, $E_{\text {kinetic }}$ is the kinetic energy, $E_{\text {potential }}$ is the elastic potential energy and $E_{\text {dissipated }}$ is the dissipated energy due to damping effects. Thus, the lowest possible value of dynamic pull-in voltage can be obtained by neglecting the damping effects. Consider the first term of eigen function expansion, the nonlinear motion equation without damped system is expressed as

$$
\ddot{q}_{1}(\tau)+\psi_{5}\left(q_{1}(\tau)\right)^{4}+\psi_{4}\left(q_{1}(\tau)\right)^{3}+\psi_{3}\left(q_{1}(\tau)\right)^{2}+\psi_{2} q_{1}(\tau)+\psi_{1}=0
$$

where $\psi_{k}=\Theta_{k} / \Theta_{0}, k \in[1,5] . \phi_{1}(\xi)$ is the first mode function as

$$
\phi_{1}(\xi)=\cosh \lambda_{1} \xi-\cos \lambda_{1} \xi-\frac{\cosh \lambda_{1}-\cos \lambda_{1}}{\sinh \lambda_{1}-\sin \lambda_{1}}\left(\sinh \lambda_{1} \xi-\sin \lambda_{1} \xi\right)
$$

where $\lambda_{1}=4.73$. To determine the relation between frequencies and physical parameters of system, the perturbation technique based on He's parameter expansion method (PEM) is used to obtain the second-order approximated analytical expression of frequency [50]. From Eq. (34) with initial conditions as $q_{1}(0)=A$ and $\dot{q}_{1}(0)=0$, 
the solution of $q_{1}(\tau)$ and the relevant factors $\psi_{1}$ and 1 are expanded in series of artificial parameter $p$ as follows [51]

$$
\begin{aligned}
& q_{1}(\tau)=\sum_{k=0}^{\infty} p^{k} q_{1, \mathrm{k}}(\tau) \\
& \psi_{1}=\omega^{2}-\sum_{m=0}^{\infty} p^{m} \chi_{m} \\
& 1=p \delta_{1}+p^{2} \delta_{2}+\cdots
\end{aligned}
$$

Substituting Eqs.(36) and (37a, b) into Eq.(34) and equating the terms with the identical powers of $p$ lead to

$$
\begin{aligned}
& p^{0}: \ddot{q}_{1,0}(\tau)+\omega^{2} q_{1,0}(\tau)=0, \quad q_{1,0}(0)=A, \quad \dot{q}_{1,0}(0)=0, \\
& p^{1}: \ddot{q}_{1,1}(\tau)+\omega^{2} q_{1,1}(\tau)=\chi_{1} q_{1,0}(\tau)-\delta_{1}\left[\psi_{2} q_{1,0}^{2}(\tau)+\psi_{3} q_{1,0}^{3}(\tau)+\psi_{4} q_{1,0}^{4}(\tau)+\psi_{0}\right], \\
& q_{1,1}(0)=0, \quad \dot{q}_{1,1}(0)=0 \\
& p^{2}: \quad \ddot{q}_{1,2}(\tau)+\omega^{2} q_{1,2}(\tau)=\chi_{1} q_{1,1}(\tau)+\chi_{2} q_{1,0}(\tau)-\delta_{1} q_{1,1}(\tau)\left[2 \psi_{2} q_{1,0}(\tau)+3 \psi_{3} q_{1,0}^{2}(\tau)\right. \\
&\left.\quad+4 \psi_{4} q_{1,0}^{3}(\tau)\right]-\delta_{2}\left[\psi_{2} q_{1,0}^{2}(\tau)+\psi_{3} q_{1,0}^{3}(\tau)+\psi_{4} q_{1,0}^{4}(\tau)+\psi_{0}\right] \\
& \\
& q_{1,2}(0)=0, \quad \dot{q}_{1,2}(0)=0
\end{aligned}
$$

Note that when $p \rightarrow 0$, Eq. (34) turns to a linear differential equation so that the approximated solution can be computed for $p=1$. The solution of the first equation is $q_{1,0}(\tau)=A \cos (\omega \tau)$ and substituting this result into Eq. (38b) generates

$$
\begin{array}{r}
\ddot{q}_{1,1}(\tau)+\omega^{2} q_{1,1}(\tau)=\left(\chi_{1} A-\frac{3}{4} \delta_{1} \psi_{3} A^{3}\right) \cos (\omega \tau)-\frac{1}{2} \delta_{1}\left(\psi_{4} A^{4}+\psi_{2} A^{2}\right) \cos (2 \omega \tau) \\
-\frac{1}{4} \delta_{1} \psi_{3} A^{3} \cos (3 \omega \tau)-\frac{1}{8} \delta_{1} \psi_{4} A^{4} \cos (4 \omega \tau)-\frac{1}{2} \delta_{1} \psi_{2} A^{2}-\frac{3}{8} \delta_{1} \psi_{4} A^{4}-\delta_{1} \psi_{0}
\end{array}
$$

where that the coefficient of term $\cos (\omega \tau)$ of the secular terms should be zero results in $\chi_{1}=\frac{3}{4} \delta_{1} \psi_{3} A^{2}$. Then solving the updated Eq. (39) for $q_{1,1}(\tau)$ gives the second-order approximated solution, as follows 


$$
\begin{aligned}
q_{1,1}(\tau)= & \frac{1}{480 \omega^{2}}\left[\left(96 \psi_{4} A^{4}+160 \psi_{2} A^{2}-15 \psi_{3} A^{3}+480 \psi_{0}\right) \cos (\omega \tau)\right. \\
& +\left(80 \psi_{4} A^{4}+80 \psi_{2} A^{2}\right) \cos (2 \omega \tau)+15 \psi_{3} A^{3} \cos (3 \omega \tau) \\
& \left.+4 \psi_{4} A^{4} \cos (4 \omega \tau)-\left(480 \psi_{0}+180 \psi_{4} A^{4}+240 \psi_{2} A^{2}\right)\right]
\end{aligned}
$$

Eqs. (38a) and (38b) for two terms approximation of series in terms of $p$, and for $p=1$ leads to relevant parameters as $\delta_{1}=1, \delta_{2}=0$ and $\chi_{2}=\omega^{2}-p \chi_{1}-\psi_{1}$. Substituting the parameters into the right-hand side of Eq. (38c) and eliminating the secular terms lead to the second-order approximated relation between frequency and amplitude, as follows

$$
\begin{gathered}
\omega^{4}-\left(\psi_{1}+\frac{3}{4} \psi_{3} A^{2}\right) \omega^{2}+2 \psi_{0} \psi_{2}+\frac{5}{6} \psi_{2}^{2} A^{2}-\frac{3}{2} \psi_{0} \psi_{3} A-\frac{1}{2} \psi_{2} \psi_{3} A^{3} \\
+\frac{3}{128} \psi_{3}^{2} A^{4}+3 \psi_{0} \psi_{4} A^{2}+\frac{7}{4} \psi_{2} \psi_{4} A^{4}-\frac{3}{10} \psi_{3} \psi_{4} A^{5}+\frac{63}{80} \psi_{4}^{2} A^{6}=0
\end{gathered}
$$

\section{Results and discussion}

\subsection{Validation of perturbation approach}

In this section, the validity of analytical model and perturbation approach is verified by comparing the results with the experiment and numerical methods from literatures. The parameters of a clamped-clamped microbeam are listed in Table 1 [52], and the corresponding dynamic pull-in voltage are compared in Table 2. The dynamic pull-in voltage by perturbation approach well agree with the results obtained by experiment [52], Displacement Iteration Pull-In Extraction (DIPIE algorithm) [53], Energy Balance Method (EBM) [54] and Finite Element Method (FEM) [55].

Also, based on the parameters for dynamic pull-in experiment of microbeam with residual axial loads in Table 3 [56] the normalized frequencies of different microbeam lengths are compared among the current perturbation approach, experiment [56], Ritz method [57], Differential quadrature method (DQM) [58] and homotopy analysis method (HAM) [59] in Table 4. It is seen from Table 4 that the present approach can accurately predict the frequency of electrostatically actuated microbeam. 
Furthermore, the convergence of perturbation approach is investigated by comparing the normalized deflection of UD-CNTRC microbeam with piezoelectric layer subjected to different electrostatic voltage and piezoelectric voltage with results calculated by the Runge-Kutta method, where $V_{c n t}=0.12, V_{e}=8, V_{p}=-1$, $A=0.5, \Delta T=200 \mathrm{~K}, \mu=0.01, \eta=0.01, \alpha_{4}=10$, shown as in Figs. 4(a, b), respectively. The accuracy of perturbation approach keep well as the external excitation loads alter and thus can be utilized to predict the dynamic behaviors of FGCNTs microbeam with piezoelectric layer.

\subsection{Nonlocal Stress and strain gradient coupling effects}

Table 5 lists the temperature-dependent material properties of CNTs and silicon at the temperature of $300 \mathrm{~K}$ (room temperature), $400 \mathrm{~K}, 500 \mathrm{~K}, 600 \mathrm{~K}$ and $700 \mathrm{~K}[60,61]$, where the elastic modulus of $[1 \overline{1} 0]$ direction silicon is used to perform data fitting to obtain the values of corresponding temperature conditions. The temperature-dependent elastic modulus of silicon satisfy the relationship of $E_{m}=(175.639-0.01987 T) \mathrm{MPa}$, where $T=T_{0}+\Delta T$ and $T_{0}=300 \mathrm{~K}$. Table 6 gives the size-dependent efficient parameters of CNTs determined by MD simulation and the rule of mixture with scale effect $[34,35]$. The physical properties of piezoelectric material are regarded to be independent of temperature. The parameters of FGCNTs reinforced microbeam and piezoelectric layer are considered as $E_{p}=78.6 \mathrm{GPa}$, $d_{31}=-123 \times 10^{-12} \mathrm{~m} / \mathrm{V}, h_{e}=1 \mu \mathrm{m}, h_{p}=0.1 \mu \mathrm{m}, L=25 h_{e}, g=0.3 h_{e}$ and $b=4.5 h_{e}$, respectively.

Fig. 4 depicts the variation of frequency under electrostatic voltage as a function of the piezoelectric excitation voltage. Note that the positive and negative piezoelectric voltages have extinct impacts on dynamic pull-in voltage. The dynamic pull-in voltage and frequency decrease as the piezoelectric voltage grows. The dynamic pull-in voltage can be tuned or adjusted by piezoelectric voltage according to the 
specific requirement of microbeam-based device.

Figs. 5(a, b) show the influence of excitation voltage exerted on piezoelectric layer on normalized frequency as a function of nonlocal stress gradient and strain gradient parameters, respectively. The increment of nonlocal stress gradient parameter decreases the frequency whereas the frequency increases as the strain gradient parameter increases. The increment of excitation voltage exerted on piezoelectric layer degrades the frequency of microbeam for both stress and strain gradient parameters.

Figs. 6(a, b) show the influence of geometrical distribution of CNTs on frequency under different controlling voltages exerted on piezoelectric layer. The frequency of UD-CNTsRC type micro-beam is lower than that of FGX-CNTsRC type but larger than that of FGO-CNTsRC, FGA-CNTsRC and FGV-CNTsRC types with identical stress and strain gradient parameters. The FGX-CNTsRC microbeam has superior stiffness to other four types subjected to electrostatic and dispersion forces. In addition, by increasing the controlling voltages exerted on piezoelectric layer, the frequency of FGX-CNTsRC microbeam significantly decreases. The stiffness of FGA-CNTsRC and FGV-CNTsRC microbeam are weaker, and thus their frequencies are lower than other three types of microbeam.

Figs. 7(a, b) depict that the normalized frequency increase with volume fraction of CNTs for both of the stress and strain gradient parameters, while the frequency declines with increase of stress gradient parameter but enhances with increment of strain gradient parameter. It is seen from Figs. 7(a, b) that the influence of volume fraction of CNTs on the normalized frequency is dependent on nonlocal models. Figs. 8(a, b) display the influence of variation of environmental temperature on frequency is obvious and the frequency decreases as the change of temperature increase for stress and strain gradient parameters, where the influence of variation of environmental temperature on frequency is dependent on nonlocal models.

Fig. 9 shows the comparison of thermal Casimir force derived by plasma model and Drude model considering thermal fluctuation with different separation distance. It is seen that at the same temperature, the values of thermal Casimir force induced by 
plasma model are larger than those of Drude model. The temperature-dependence of thermal Casimir force is more significant at higher temperature and wider initial gap.

Figs. 10 (a, b) compare the influences of quantum and thermal Casimir force on the normalized frequency, respectively. The previous studies on Casimir effect on micro-/nano-scale microbeam was not able to consider the thermal fluctuation effect at the finite temperature environment. Actually, the frequency of microbeam is impacted by quantum Casimir force in connection with thermal Casimir forceinduced by thermal fluctuation at the finite temperature. Compared to quantum Casimir force, the increment of the thermal Casimir force further decreases the normalized frequency. The previous results overestimate the frequency of microbeam subjected to electrostatic and quantum Casimir forces.

Fig.11 depicts the coupling effects of nonlocal stress and strain gradient parameters on the frequency of FGCNTs reinforced microbeam with piezoelectric layer, where UD-CNTsRC, $V_{c n t}=0.17, V_{e}=5, V_{p}=1, A=0.2, \Delta T=200 \mathrm{~K}, \quad \alpha_{4}=1$ and $\beta_{1}=\beta_{2}=1$. The frequency declines with the increase of nonlocal stress gradient parameter while increases with increment of strain gradient parameter. As a result, the size dependency of dynamic microbeam layer is determined by the coupling impacts of two intrinsic material length parameters.

\subsection{Dynamic behaviors of damping system}

To further investigate the dynamic behaviors of microbeam with piezoelectric layer near the critical pull-in domain, the time history response and phase plane without damping effects are illustrated in Figs. 12(a, b), respectively. As can be observed, the deflection increases with increment of electrostatic voltage before the pull-in occurs; when the voltage increases to the pull-in value, $V_{e}=8.56$ in Fig. 12(a), the dynamic pull-in happens and the microbeam with piezoelectric layer loses its structural stability and collapse onto the ground electrode. From the phase diagram in Fig. 12(b), it is seen that at the lower voltage, the dynamic system presents periodic motion 
around the stable center point. When the voltage approaches to the pull-in value, the trajectory gets closer to the unstable saddle node. By increasing the voltage to the pull-in value, the system becomes dynamically unstable and sticks to the ground electrode.

Figs. 13(a, b) show the time history and phase plane of dynamic system with damping effects, respectively. From Fig. 13(a), it is seen that the magnitude of deflection gradually decreases due to the damping effects before the pull-in state, and when the applied voltage increases to dynamic pull-in value $\left(V_{e}=8.64\right)$ the amplitude becomes divergent and the pull-in instability occurs, which leads the microbeam to collapse onto the substrate. Note that the damping effects increase the values of dynamic pull-in voltage, because the structural and viscous damping dissipate a part of injected electrostatic energy of dynamic system. In Fig. 13(b), before the pull-in instability, the phase plane has one stable center; owing to the damping effects, the trajectory gradually converges into the center point. The dynamic system has homoclinic orbit that originates from unstable branch and returns to the saddle node at the stable one. At the dynamic pull-in voltage, the trajectory loses its stable center and becomes divergent. After the voltage beyond the pull-in value, the system becomes unstable and touches the substrate.

Figs. 14(a, b) demonstrate the coupling influences of nonlocal stress and strain gradient parameters on dynamic behaviors of damping system at the pull-in state. It is seen from Fig. 14(a) that nonlocal stress gradient parameter extends the pull-in time while the pull-in time obviously shortens with the increment of strain gradient parameter. The pull-in deflection decreases as the stress gradient parameter increases while the pull-in amplitude magnifies by increasing strain gradient parameter. Moreover, in Fig. 14(b), the velocity of dynamic system increases as the strain gradient parameter whereas the velocity reduces with increase of the stress gradient parameter.

\section{Conclusion}


A unifying nonlocal stress and strain gradient model is presented to study size-dependent dynamic characteristics of electrostatically actuated FGCNTs reinforced microbeam with piezoelectric layer. The tuning effect of piezoelectric layer on pull-in instability, the quantum and thermal Casimir force and damping effect are also considered. Main conclusions are list as follows

1) The frequency and dynamic pull-in voltage of microbeam with piezoelectric layer decrease with the increase of excitation voltage exerted on piezoelectric layer. The dynamic pull-in voltage of microbeam can be tuned by piezoelectric effect.

2) The size dependency of microbeam is determined by coupling effects of nonlocal stress and strain gradient parameters. The frequency declines with the increment of stress gradient while increases with the growth of strain gradient.

3) The frequency of FGX-CNTsRC type microbeam is larger than that of UD-CNTsRC, FGO-CNTsRC, FGA-CNTsRC and FGV-CNTsRC because of FGX-CNTsRC microbeam having superior stiffness characteristics under electrostatic and dispersion forces. The stiffness of FGA-CNTsRC and FGV-CNTsRC microbeam are weaker, and thus their frequencies are lower than other three types of microbeam.

4) The quantum and thermal Casimir effect cannot be ignored on investigating dynamic behaviors of microbeam at finite temperature filed. The previous studies overestimate the frequency of electrostatically actuated microbeam. The frequency declines with increase of quantum and thermal Casimir forces.

5) The pull-in voltage of damping system is higher than that of undamped system due to dissipative impacts of damping. The pull-in deflection and velocity of microbeam decrease with the growth of stress gradient parameter but increase with the increment of strain gradient parameter.

\section{Appendix A}

The associated coefficients of the normalized nonlocal governing equation of motion are expressed as follows 


$$
\begin{aligned}
\Theta_{0}= & K_{4} \int_{0}^{1} \phi_{i}(\xi) \phi_{j}(\xi) d \xi-\mu K_{4} \int_{0}^{1} \phi_{i}(\xi) \phi_{j}^{I I}(\xi) d \xi \\
\Theta_{1}= & -\left[V_{e}^{2}(1+\gamma)+(1-n) \alpha_{3}+n \alpha_{4}+n \beta_{1}+n \beta_{2}\right] \int_{0}^{1} \phi_{n}(\xi) d \xi \\
\Theta_{2}= & {\left[K_{3}-\mu K_{2}\left(N^{T}+N^{P}\right)\right] \int_{0}^{1} \phi_{i}(\xi) \phi_{j}^{I V}(\xi) d \xi } \\
& -\eta K_{3} \int_{0}^{1} \phi_{i}(\xi) \phi_{j}^{V I}(\xi) d \xi+K_{2}\left(N^{T}+N^{P}\right) \int_{0}^{1} \phi_{i}(\xi) \phi_{j}^{I I}(\xi) d \xi \\
& -\left[V_{e}^{2}(2+\gamma)+3(1-n) \alpha_{3}+4 n \alpha_{4}+3 n \beta_{2}\right]\left[\int_{0}^{1} \phi_{i}(\xi) \phi_{j}(\xi) d \xi-\mu \int_{0}^{1} \phi_{i}(\xi) \phi_{j}^{I I}(\xi) d \xi\right] \\
\Theta_{3}= & -\left[V_{e}^{2}(3+\gamma)+6(1-n) \alpha_{3}+10 n \alpha_{4}+6 n \beta_{2}\right]\left[\int_{0}^{1} \phi_{i}(\xi) \phi_{j}(\xi) \phi_{k}(\xi) d \xi\right. \\
& \left.-2 \mu \int_{0}^{1} \phi_{i}(\xi) \phi_{j}^{I}(\xi) \phi_{k}^{I}(\xi) d \xi-2 \mu \int_{0}^{1} \phi_{i}(\xi) \phi_{j}(\xi) \phi_{k}^{I I}(\xi) d \xi\right] \\
\Theta_{4}= & K_{1} \Gamma\left[\phi_{i}(\xi), \phi_{j}(\xi)\right]\left(\mu \int_{0}^{1} \phi_{k}(\xi) \phi_{m}^{I V}(\xi) d \xi-\int_{0}^{1} \phi_{k}(\xi) \phi_{m}^{I I}(\xi) d \xi\right) \\
& -\left[V_{e}^{2}(4+\gamma)+10(1-n) \alpha_{3}+20 n \alpha_{4}+10 n \beta_{2}\right]\left[\int_{0}^{1} \phi_{i}(\xi) \phi_{j}(\xi) \phi_{k}(\xi) \phi_{m}(\xi) d \xi\right. \\
& \left.-6 \mu \int_{0}^{1} \phi_{i}(\xi) \phi_{j}(\xi) \phi_{k}^{I}(\xi) \phi_{m}^{I}(\xi) d \xi-3 \mu \int_{0}^{1} \phi_{i}(\xi) \phi_{j}(\xi) \phi_{k}(\xi) \phi_{m}^{I I}(\xi) d \xi\right] \\
& -\mu \hat{C}_{v d} \int_{0}^{1} \phi_{i}(\xi) \phi_{j}^{I I}(\xi) d \xi \\
\Theta_{5}= & -\left[V_{e}^{2}(5+\gamma)+15(1-n) \alpha_{3}+35 n \alpha_{4}+15 n \beta_{2}\right]\left[\int_{0}^{1} \phi_{i}(\xi) \phi_{j}(\xi) \phi_{k}(\xi) \phi_{m}(\xi) \phi_{r}(\xi) d \xi\right. \\
& \left.-12 \mu \int_{0}^{1} \phi_{i}(\xi) \phi_{j}(\xi) \phi_{k}(\xi) \phi_{m}^{I}(\xi) \phi_{r}^{I}(\xi) d \xi-4 \mu \int_{0}^{1} \phi_{i}(\xi) \phi_{j}(\xi) \phi_{k}(\xi) \phi_{m}(\xi) \phi_{r}^{I I}(\xi) d \xi\right] \\
\Theta_{6}= & \mathcal{O}_{s d} \int_{0}^{1} \phi_{i}(\xi) \phi_{j}^{I}(\xi) d \xi-\mu C_{s d} \int_{0}^{1} \phi_{i}(\xi) \phi_{j}^{I I I}(\xi) d \xi+C_{v d}^{1} \phi_{0}(\xi) \phi_{j}(\xi) d \xi \\
&
\end{aligned}
$$

where $\Gamma\left[\phi_{k}(\xi, \tau), \phi_{m}(\xi, \tau)\right]=\int_{0}^{1} \frac{\partial \phi_{k}}{\partial \xi} \frac{\partial \phi_{m}}{\partial \xi} d \xi$

\section{Acknowledge}

The authors acknowledge financial support from National Science Foundation of China under Number 11172165.

\section{References}

[1] S.E. Lyshevski, MEMS and NEMS: systems, devices, and structures: CRC Press; 2013.

[2] H. Mobki, G. Rezazadeh, M. Sadeghi, F. Vakili-Tahami, M.-M. Seyyed-Fakhrabadi, A comprehensive study of stability in an electro-statically actuated micro-beam, Int J Non Linear Mech 48(2013) 78-85.

[3] T. Mousavi, S. Bornassi, H. Haddadpour, The effect of small scale on the pull-in instability of 
nano-switches using DQM, Int J Solids Struct 50(2013) 1193-202.

[4] D.I. Caruntu, I. Martinez, Reduced order model of parametric resonance of electrostatically actuated MEMS cantilever resonators, Int J Non Linear Mech 66(2014) 28-32.

[5] W.D. Yang, X. Wang, C.Q. Fang, G. Lu, Electromechanical coupling characteristics of carbon nanotube reinforced cantilever nano-actuator, Sens Actuators, A 220(2014) 178-87.

[6] W. Yang, X. Wang, C. Fang, Pull-in instability of carbon nanotube-reinforced nano-switches considering scale, surface and thermal effects, Composites Part B: Engineering, 82(2015) 143-51.

[7] W.-M. Zhang, H. Yan, Z.-K. Peng, G. Meng, Electrostatic pull-in instability in MEMS/NEMS: A review, Sens Actuators, A 214(2014) 187-218.

[8] K. Choi, N. Muhammad, M. Rehmani, D. Kim, Hybrid piezo-electrostatic inkjet head for printed electronics, Proc Inst Mech Eng Part C J Mech Eng Sci 226(2012) 842-57.

[9] H. Raeisifard, M.N. Bahrami, A. Yousefi-Koma, H.R. Fard, Static characterization and pull-in voltage of a micro-switch under both electrostatic and piezoelectric excitations, European Journal of Mechanics-A/Solids, 44(2014) 116-24.

[10] C.-C. Liu, C.-H. Liu, Analysis of nonlinear dynamic behavior of electrically actuated micro-beam with piezoelectric layers and squeeze-film damping effect, Nonlinear Dyn 77(2014) 1349-61.

[11] Y. Xiao, B. Wang, S. Zhou, Pull-in voltage analysis of electrostatically actuated MEMS with piezoelectric layers: A size-dependent model, Mechanics Research Communications, 66(2015) 7-14.

[12] C. Chen, H. Hu, L. Dai, Nonlinear behavior and characterization of a piezoelectric laminated microbeam system, Communications in Nonlinear Science and Numerical Simulation, 18(2013) 1304-15.

[13] S. Azizi, G. Rezazadeh, M.-R. Ghazavi, S.E. Khadem, Stabilizing the pull-in instability of an electro-statically actuated micro-beam using piezoelectric actuation, Applied Mathematical Modelling, 35(2011) 4796-815.

[14] J. Zou, Z. Marcet, A.W. Rodriguez, M.T. Reid, A.P. McCauley, Kravchenko, II, et al., Casimir forces on a silicon micromechanical chip, Nat Commun, 4(2013) 1845.

[15] H.B. Chan, V.A. Aksyuk, R.N. Kleiman, D.J. Bishop, F. Capasso, Quantum mechanical actuation of microelectromechanical systems by the Casimir force, Science, 291(2001) 1941-4.

[16] K. Milton, The Casimir force: Feeling the heat, Nat Phys 7(2011) 190-1.

[17] A. Sushkov, W. Kim, D. Dalvit, S. Lamoreaux, Observation of the thermal Casimir force, Nat Phys 7(2011) 230-3.

[18] Y.-G. Wang, W.-H. Lin, X.-M. Li, Z.-J. Feng, Bending and vibration of an electrostatically actuated circular microplate in presence of Casimir force, Applied Mathematical Modelling, 35(2011) 2348-57.

[19] H.M. Sedighi, F. Daneshmand, M. Abadyan, Dynamic instability analysis of electrostatic functionally graded doubly-clamped nano-actuators, Compos Struct 124(2015) 55-64.

[20] A.C. Eringen, D. Edelen, On nonlocal elasticity, Int J Eng Sci 10(1972) 233-48.

[21] R. Barretta, L. Feo, R. Luciano, Torsion of functionally graded nonlocal viscoelastic circular nanobeams, Composites Part B: Engineering, 72(2015) 217-22.

[22] F. Yang, A. Chong, D. Lam, P. Tong, Couple stress based strain gradient theory for elasticity, Int J Solids Struct 39(2002) 2731-43.

[23] S. Park, X. Gao, Bernoulli-Euler beam model based on a modified couple stress theory, J Micromech Microeng 16(2006) 2355.

[24] D. Lam, F. Yang, A. Chong, J. Wang, P. Tong, Experiments and theory in strain gradient elasticity, J Mech Phys Solids 51(2003) 1477-508. 
[25] H. Dai, L. Wang, Q. Ni, Dynamics and pull-in instability of electrostatically actuated microbeams conveying fluid, Microfluid Nanofluid 18(2015) 49-55.

[26] H.M. Sedighi, Size-dependent dynamic pull-in instability of vibrating electrically actuated microbeams based on the strain gradient elasticity theory, Acta Astronaut 95(2014) 111-23.

[27] C. Lim, G. Zhang, J. Reddy, A higher-order nonlocal elasticity and strain gradient theory and its applications in wave propagation, J Mech Phys Solids 78(2015) 298-313.

[28] L. Li, Y. Hu, L. Ling, Flexural wave propagation in small-scaled functionally graded beams via a nonlocal strain gradient theory, Compos Struct 133(2015) 1079-92.

[29] L. Li, Y. Hu, Buckling analysis of size-dependent nonlinear beams based on a nonlocal strain gradient theory, Int J Eng Sci 97(2015) 84-94.

[30] K. Liew, Z. Lei, L. Zhang, Mechanical analysis of functionally graded carbon nanotube reinforced composites: A review, Compos Struct 120(2015) 90-7.

[31] F. Ebrahimi, E. Salari, Thermo-mechanical vibration analysis of nonlocal temperature-dependent FG nanobeams with various boundary conditions, Composites Part B: Engineering, 78(2015) 272-90.

[32] Q. Jiang, X. Wang, Y. Zhu, D. Hui, Y. Qiu, Mechanical, electrical and thermal properties of aligned carbon nanotube/polyimide composites, Composites Part B: Engineering, 56(2014) 408-12.

[33] F. Gardea, D.C. Lagoudas, Characterization of electrical and thermal properties of carbon nanotube/epoxy composites, Composites Part B: Engineering, 56(2014) 611-20.

Mathematical Modelling, 38(2014) 2934-45.

[34] H.-S. Shen, Nonlinear bending of functionally graded carbon nanotube-reinforced composite plates in thermal environments, Compos Struct 91(2009) 9-19.

[35] L.-L. Ke, J. Yang, S. Kitipornchai, Nonlinear free vibration of functionally graded carbon nanotube-reinforced composite beams, Compos Struct 92(2010) 676-83.

[36] N. Wattanasakulpong, V. Ungbhakorn, Analytical solutions for bending, buckling and vibration responses of carbon nanotube-reinforced composite beams resting on elastic foundation, Comput Mater Sci 71(2013) 201-8.

[37] M. Mirzaei, Y. Kiani, Snap-through phenomenon in a thermally postbuckled temperature dependent sandwich beam with FG-CNTRC face sheets, Compos Struct 134(2015) 1004-13.

[38] M. Mirzaei, Y. Kiani, Nonlinear free vibration of temperature-dependent sandwich beams with carbon nanotube-reinforced face sheets, Acta Mech (2016) 1-16.

[39] J. Jam, Y. Kiani, Low velocity impact response of functionally graded carbon nanotube reinforced composite beams in thermal environment, Compos Struct 132(2015) 35-43.

[40] Y. Kiani, Thermal postbuckling of temperature-dependent sandwich beams with carbon nanotube-reinforced face sheets, J Therm Stresses 39(2016) 1098-110.

[41] M. Rafiee, J. Yang, S. Kitipornchai, Large amplitude vibration of carbon nanotube reinforced functionally graded composite beams with piezoelectric layers, Compos Struct 96(2013) 716-25.

[42] S.K. Ha, Admittance matrix of asymmetric piezoelectric bimorph with two separate electrical ports under general distributed loads, Ultrasonics, Ferroelectrics, and Frequency Control, IEEE Transactions on, 48(2001) 976-84.

[43] E. Lifshitz, The theory of molecular attractive forces between solids, (1956).

[44] I. Brevik, J.S. Høye, Temperature dependence of the Casimir force, Eur J Phys 35(2014) 015012.

[45] J.N. Reddy, S. El-Borgi, J. Romanoff, Non-linear analysis of functionally graded microbeams using Eringen's non-local differential model, Int J Non Linear Mech 67(2014) 308-18.

[46] J.N. Reddy, Nonlocal theories for bending, buckling and vibration of beams, Int J Eng Sci 45(2007) 
288-307.

[47] G. Rezazadeh, A. Tahmasebi, M. Zubstov, Application of piezoelectric layers in electrostatic MEM actuators: controlling of pull-in voltage, Microsyst Technol 12(2006) 1163-70.

[48] M. Mojahedi, M.T. Ahmadian, K. Firoozbakhsh, Dynamic Pull-in Instability and Vibration Analysis of a Nonlinear Microcantilever Gyroscope under Step Voltage Considering Squeeze Film Damping, International Journal of Applied Mechanics, 05(2013) 1350032.

[49] L.-D. Liao, P.C. Chao, C.-W. Huang, C.-W. Chiu, DC dynamic and static pull-in predictions and analysis for electrostatically actuated clamped circular micro-plates based on a continuous model, J Micromech Microeng 20(2010) 025013.

[50] J.-H. He, Some asymptotic methods for strongly nonlinear equations, Int J Mod Phys B 20(2006) 1141-99.

[51] J.-H. He, Bookkeeping parameter in perturbation methods, International Journal of Nonlinear Sciences and Numerical Simulation, 2(2001) 257-64.

[52] R.K. Gupta, E.S. Hung, Y.-J. Yang, G. Ananthasuresh, S.D. Senturia, Pull-in dynamics of electrostatically-actuated beams, Proceedings of the Solid-State Sensor and Actuator Workshop1996.

[53] O. Bochobza-Degani, D. Elata, Y. Nemirovsky, An efficient DIPIE algorithm for CAD of electrostatically actuated MEMS devices, Microelectromechanical Systems, Journal of, 11(2002) 612-20.

[54] G.N. Nielson, G. Barbastathis, Dynamic pull-in of parallel-plate and torsional electrostatic MEMS actuators, Microelectromechanical Systems, Journal of, 15(2006) 811-21.

[55] P.C. Chao, C. Chiu, T.-H. Liu, DC dynamic pull-in predictions for a generalized clamped-clamped micro-beam based on a continuous model and bifurcation analysis, J Micromech Microeng 18(2008) 115008.

[56] R. Legtenberg, H.A. Tilmans, Electrostatically driven vacuum-encapsulated polysilicon resonators Part I. Design and fabrication, Sens Actuators, A 45(1994) 57-66.

[57] H.A. Tilmans, R. Legtenberg, Electrostatically driven vacuum-encapsulated polysilicon resonators: Part II. Theory and performance, Sens Actuators, A 45(1994) 67-84.

[58] J.-H. Kuang, C.-J. Chen, Dynamic characteristics of shaped micro-actuators solved using the differential quadrature method, J Micromech Microeng 14(2004) 647.

[59] M. Moghimi Zand, M.T. Ahmadian, Application of homotopy analysis method in studying dynamic pull-in instability of microsystems, Mechanics Research Communications, 36(2009) 851-8.

[60] Y. Okada, Y. Tokumaru, Precise determination of lattice parameter and thermal expansion coefficient of silicon between 300 and $1500 \mathrm{~K}$, J Appl Phys 56(1984) 314-20.

[61] C.-H. Cho, Characterization of Young's modulus of silicon versus temperature using a "beam deflection" method with a four-point bending fixture, Curr Appl Phys 9(2009) 538-45. 


\section{Capital of Figures}

Fig. 1. Schematic of different micro-/nano-scale dispersion forces: (a) van der Waals force, (b) Casimir-Polder force, (c) Casimir force at zero temperature and (d) quantum and thermal fluctuation induced Casimir force at finite temperature environment.

Fig.2. (a) Model of FGCNTs reinforced microbeam with piezoelectric layer subjected to electrostatic and intermolecular forces, and (b) Geometrical distribution of CNTs reinforcement in elastic layer.

Fig.3. Comparison of time history response under (a) electrostatic voltage and (b) piezoelectric voltage from second-order perturbation approach and numerical approach.

Fig.4. Influence of controlling voltage exerted on piezoelectric layer on dynamic pull-in voltage and fundamental frequency of FGCNTs reinforced microbeam, where UD-CNTsRC, $V_{c n t}=0.12, V_{e}=10, A=0.2, \Delta T=200 \mathrm{~K}, \mu=0.05, \eta=0.05$ and $\alpha_{4}=1$.

Figs.5 (a, b).Influence of excitation voltage exerted on piezoelectric layer on normalized frequency as the function of (a) nonlocal stress gradient and (b) strain gradient parameter $\quad\left(\right.$ FGX-CNTsRC $, V_{c n t}=0.17, V_{e}=5, A=0.2, \Delta T=200 \mathrm{~K}$, $\mu=0.05, \eta=0.05$ and $\left.\alpha_{4}=1\right)$.

Figs.6 (a, b). Influences of geometrical distribution of CNTs on frequency under controlling voltages exerted on piezoelectric layer for (a) nonlocal stress gradient and (b) strain gradient parameter $\left(V_{c n t}=0.28, V_{e}=5, A=0.2, \Delta T=200 \mathrm{~K}\right.$ and $\left.\alpha_{4}=1\right)$.

Figs.7 (a, b). Influences of volume fraction of CNTs on normalized frequency for different nonlocal models as (a) nonlocal stress gradient and (b) strain gradient parameter (FGO-CNTsRC, $V_{e}=5, A=0.2, \Delta T=200 \mathrm{~K}$ and $\alpha_{4}=1$ ).

Figs.8 (a, b). Influences of variation of environmental temperature on normalized frequency for different nonlocal models (a) nonlocal stress gradient and (b) strain 
gradient parameter (FGX-CNTsRC, $V_{c n t}=0.17, V_{e}=5, V_{p}=1, A=0.2$ and $\alpha_{4}=1$ ).

Fig. 9.Temperature-dependent thermal Casimir force based on Plasma model and Drude model under different initial gap

Figs. $10(\mathrm{a}, \mathrm{b})$. Comparison of quantum and thermal Casimir effect on normalized frequency for different nonlocal models (a) nonlocal stress gradient and (b) strain gradient parameter (FGX-CNTsRC, $V_{c n t}=0.17, V_{e}=5, V_{p}=1, A=0.2$ and $\Delta T=200 \mathrm{~K})$.

Fig. 11.Coupling effects of nonlocal stress and strain gradient parameters on normalized frequency of FGCNTs reinforced microbeam with piezoelectric layer.

Figs. $12(a, b)$. Dynamic behaviors of microbeam without damping effects near dynamic pull-in domain for (a) time story response and (b) phase plane near pull-in domain $\left(\mu=0.1, \eta=0.1, \hat{C}_{s d}=\hat{C}_{v d}=0\right)$.

Figs. $13(a, b)$. Dynamic behaviors of microbeam with damping effects near dynamic pull-in domain for (a) time story response and (b) phase plane near pull-in domain $\left(\mu=0.1, \eta=0.1, \hat{C}_{s d}=1\right.$ and $\left.\hat{C}_{v d}=1\right)$.

Figs. $14(\mathrm{a}, \mathrm{b})$.Dynamic behaviors of microbeam with damping effects at dynamic pull-in state for (a) time story response and (b) phase plane $\left(\hat{C}_{s d}=1\right.$ and $\left.\hat{C}_{v d}=1\right)$.

Table 1. Experimental parameters of dynamic pull-in for a clamped-clamped microbeam [52].

Table 2. Comparison of dynamic pull-in voltage obtained by different methods.

Table 3. Parameters of dynamic pull-in experiment with axial residual stress [56].

Table 4. Comparison of fundamental frequencies obtained by different methods.

Table 5. Temperature-dependent material property of CNTs and silicon [60, 61].

Table 6. CNTs efficient parameter of scale-dependent property for different volume fraction $[34,35]$. 
(a)

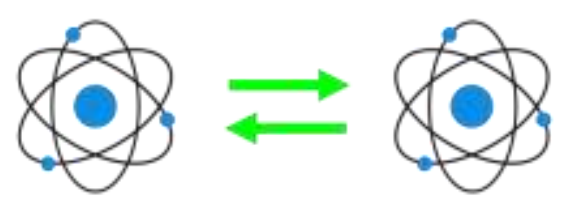

(b)
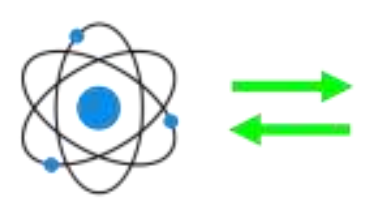

(c)
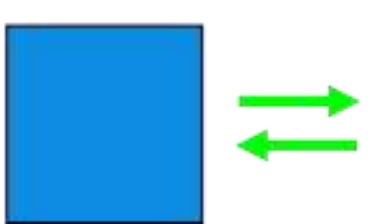

(d)

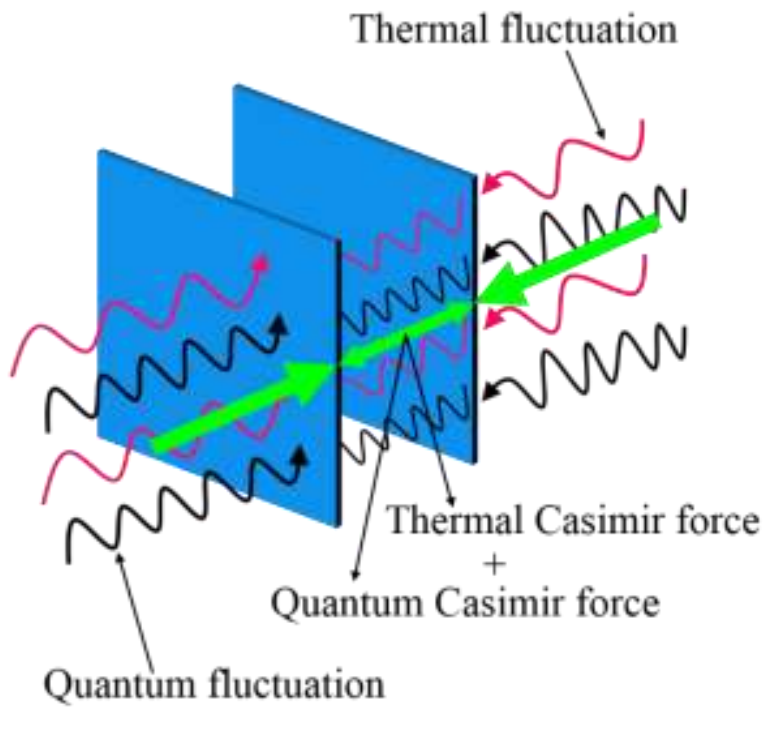

Fig. 1. Schematic of different micro-/nano-scale dispersion forces: (a) van der Waals force, (b) Casimir-Polder force, (c) Casimir force at zero temperature and (d) quantum and thermal fluctuation induced Casimir force at finite temperature environment.

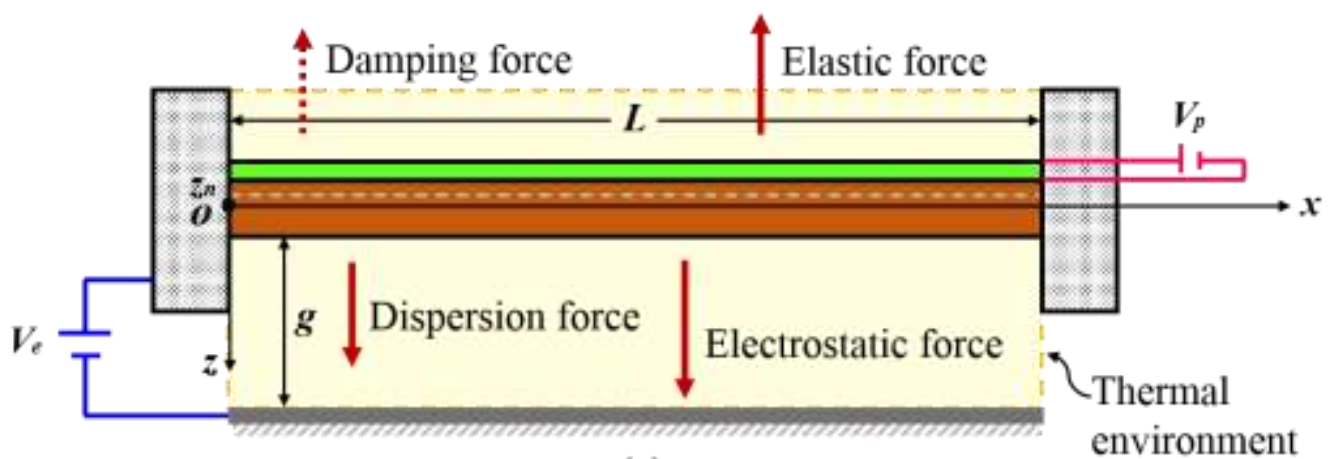

(a)

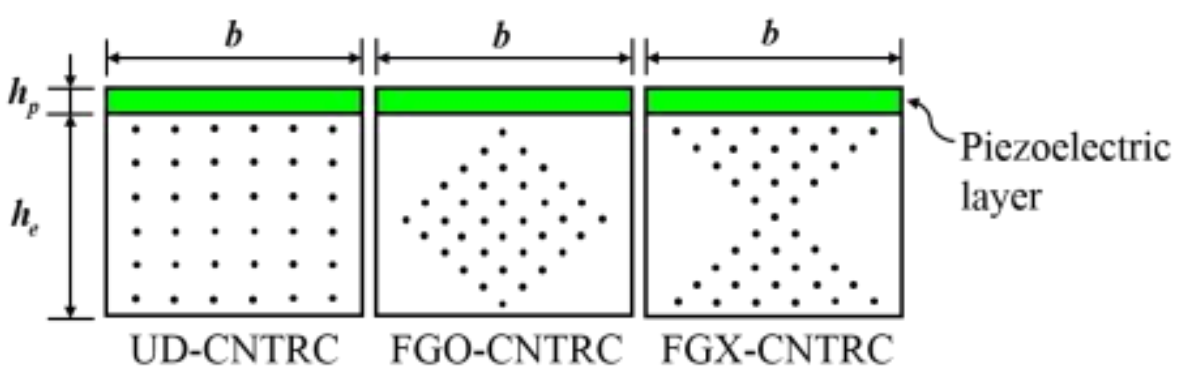

(b)

Fig.2. Model of FGCNTs reinforced microbeam with piezoelectric layer subjected to electrostatic and intermolecular forces. 

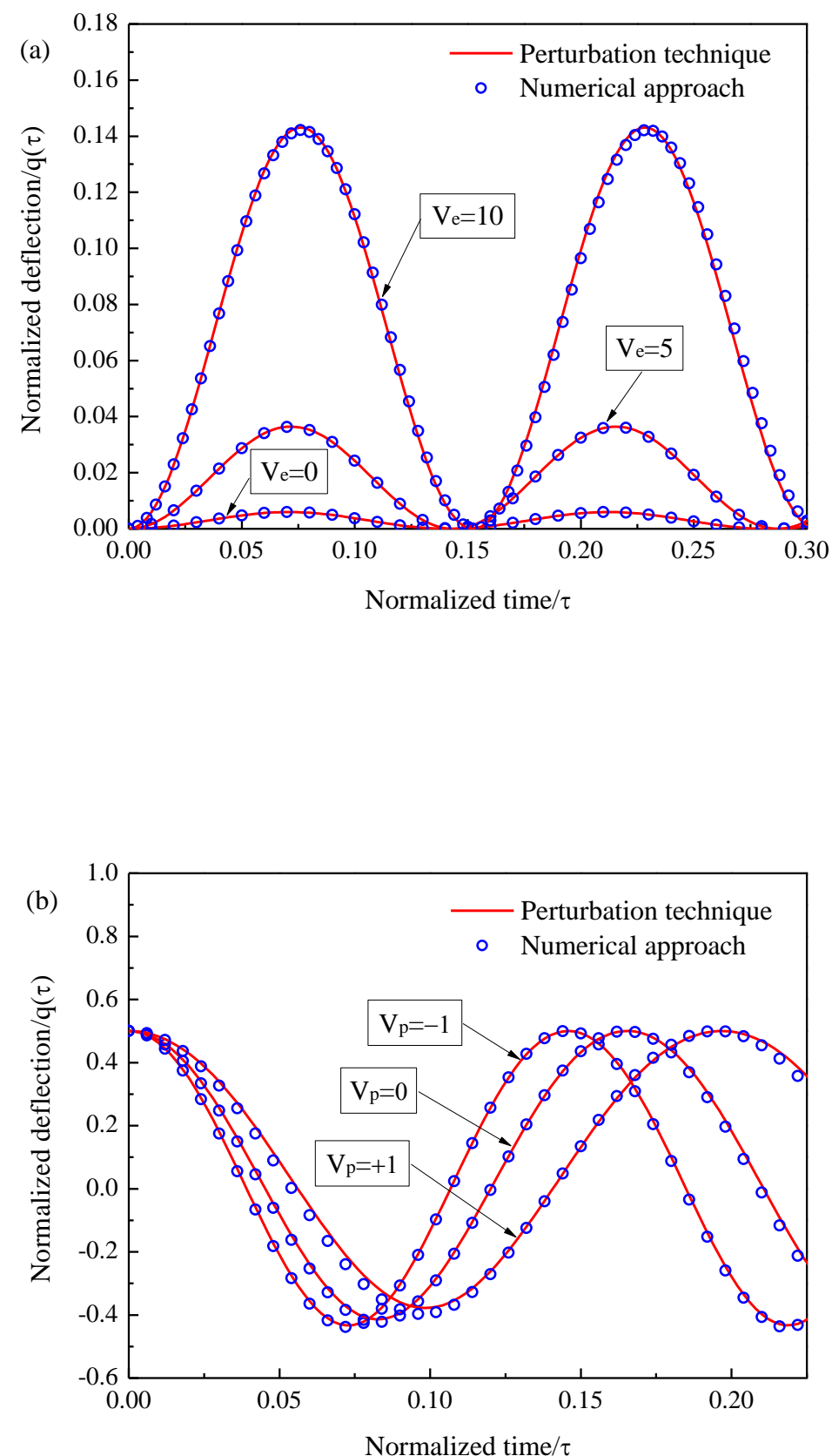

Fig.3. Comparison of time history response under (a) electrostatic voltage and (b) piezoelectric voltage from second-order perturbation approach and numerical approach. 


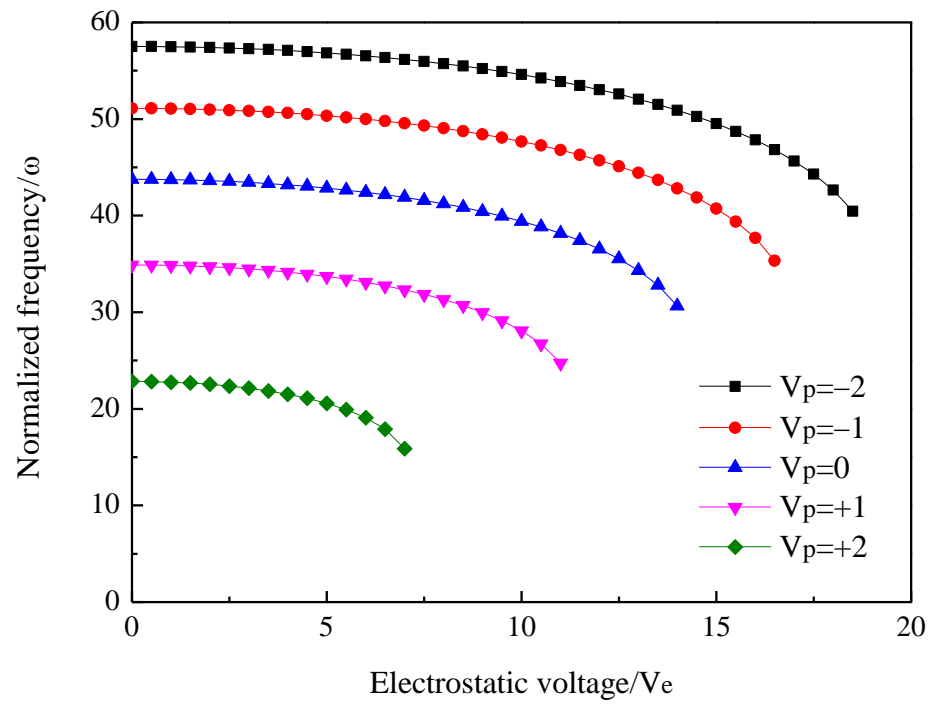

Fig.4. Influence of controlling voltage exerted on piezoelectric layer on dynamic pull-in voltage and fundamental frequency of FGCNTs reinforced microbeam, where UD-CNTsRC,

$$
V_{c n t}=0.12, V_{e}=10, A=0.2, \Delta T=200 \mathrm{~K}, \mu=0.05, \eta=0.05 \text { and } \alpha_{4}=1 .
$$

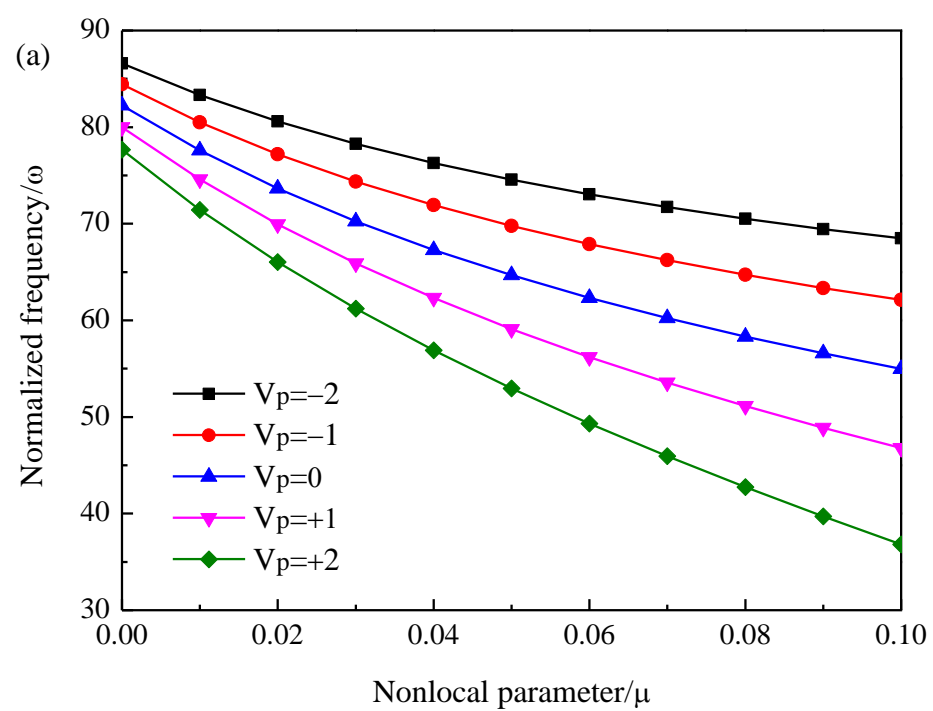




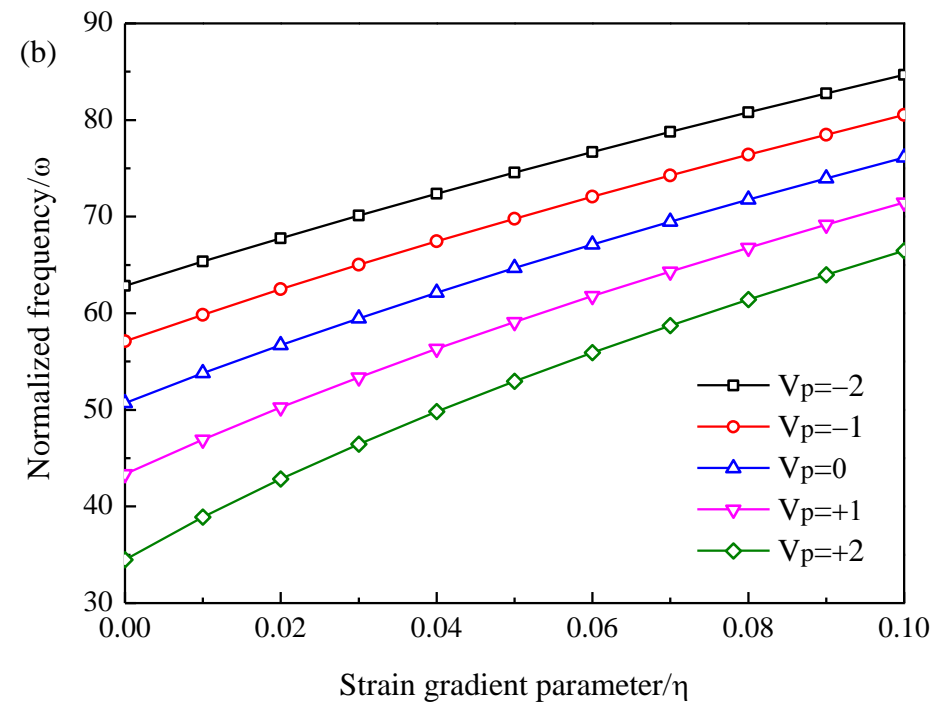

Figs.5 (a, b).Influence of excitation voltage exerted on piezoelectric layer on normalized frequency as the function of (a) nonlocal stress gradient and (b) strain gradient parameter (FGX-CNTsRC, $V_{c n t}=0.17, V_{e}=5, A=0.2, \Delta T=200 \mathrm{~K}, \mu=0.05, \eta=0.05$ and $\alpha_{4}=1$ ).

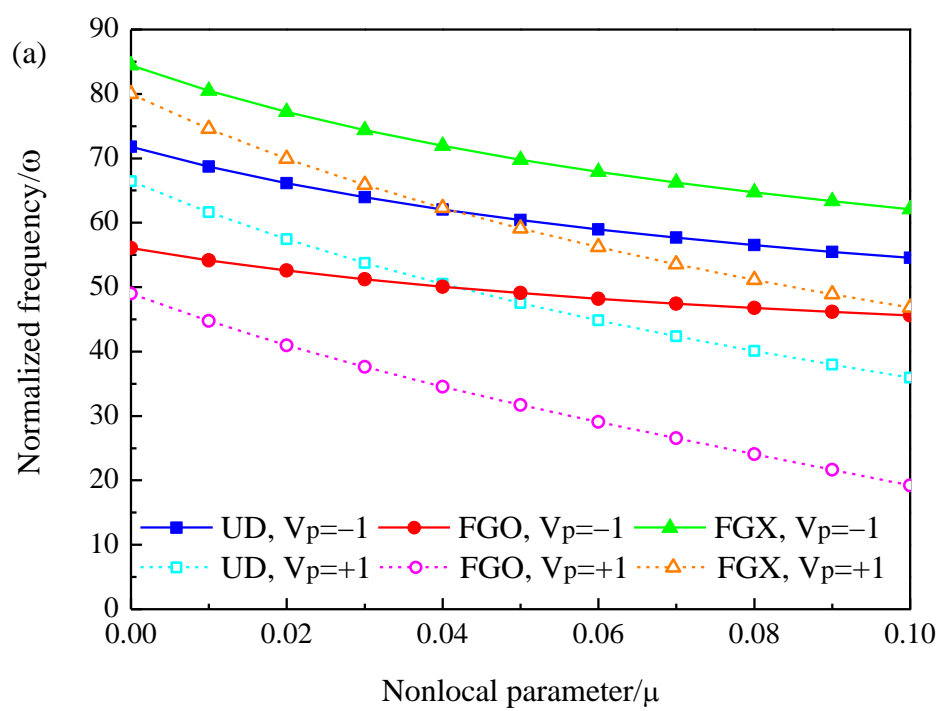




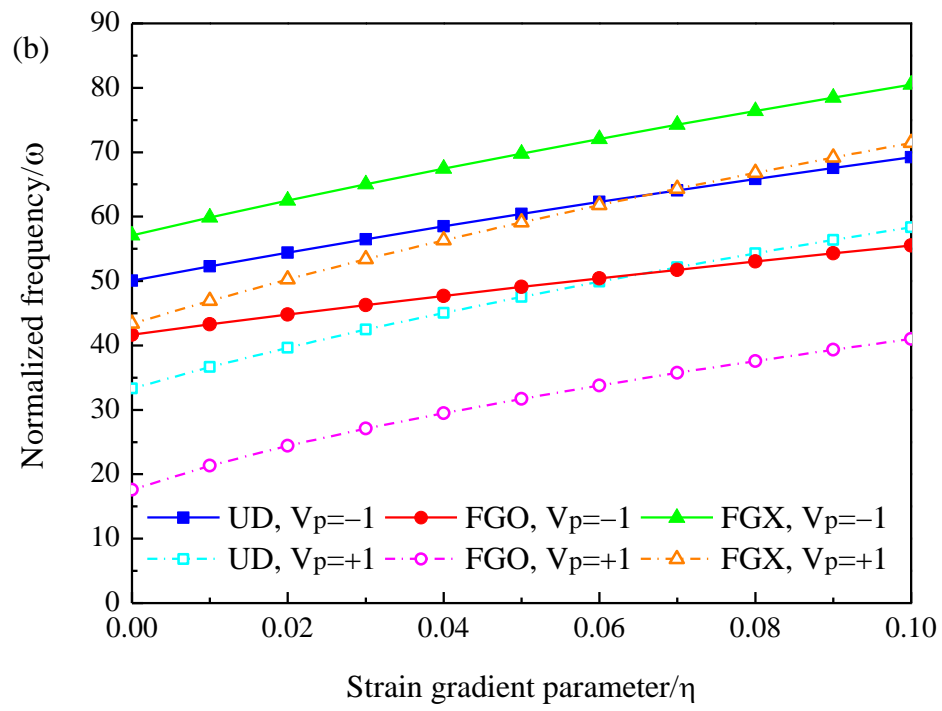

Figs.6 (a, b). Influences of geometrical distribution of CNTs on frequency under controlling voltages exerted on piezoelectric layer for (a) nonlocal stress gradient and (b) strain gradient parameter $\left(V_{c n t}=0.17, V_{e}=5, A=0.2, \Delta T=200 \mathrm{~K}\right.$ and $\left.\alpha_{4}=1\right)$.

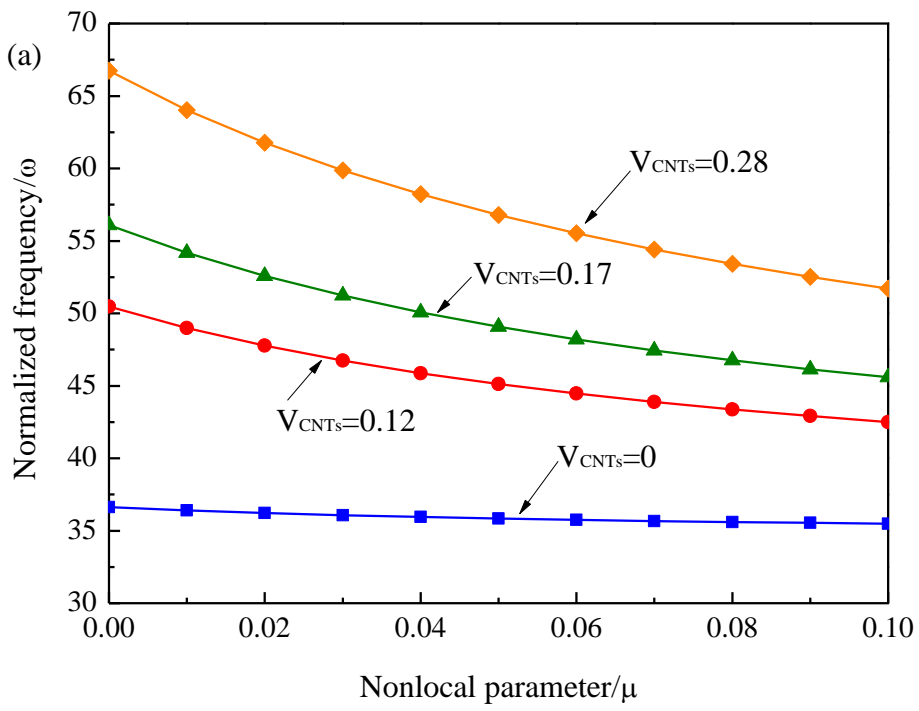




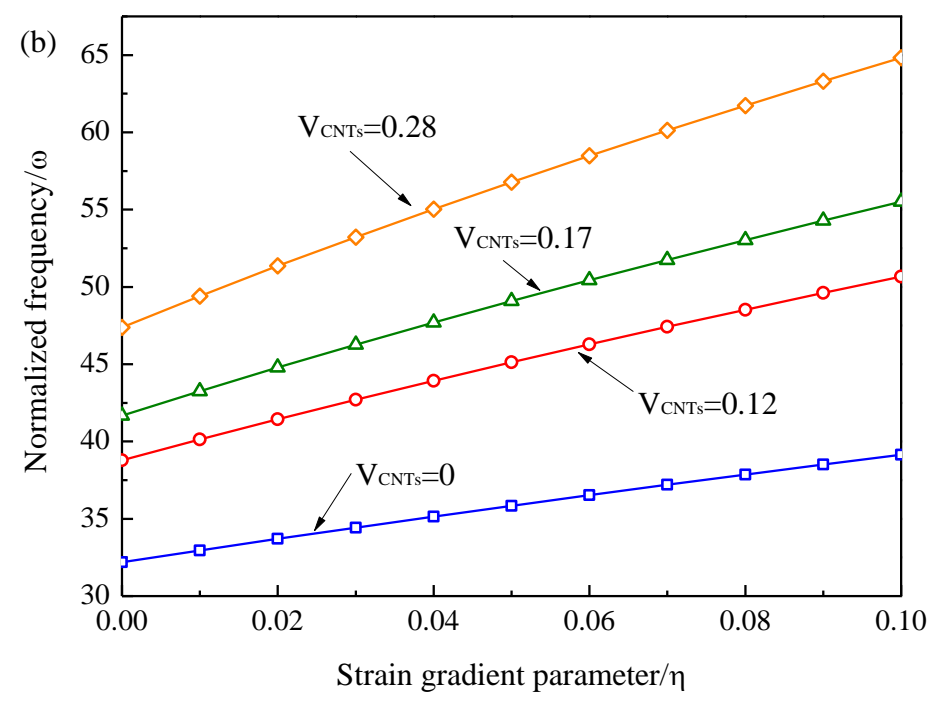

Figs.7 (a, b). Influences of volume fraction of CNTs on normalized frequency for different nonlocal models as (a) nonlocal stress gradient and (b) strain gradient parameter (FGO-CNTsRC, $V_{e}=5, A=0.2, \Delta T=200 \mathrm{~K}$ and $\alpha_{4}=1$ ).

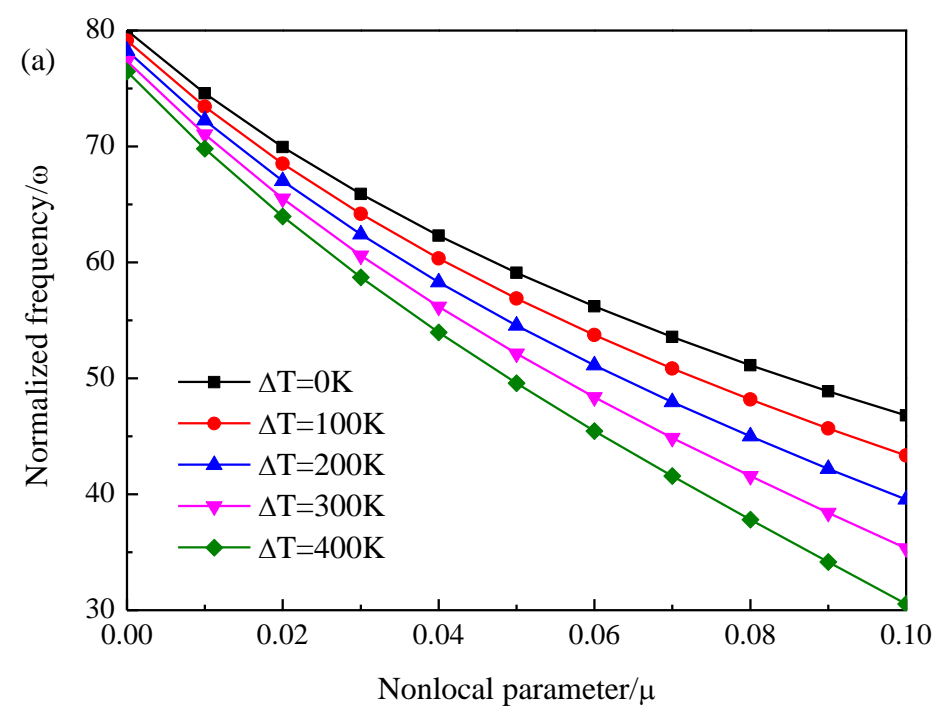




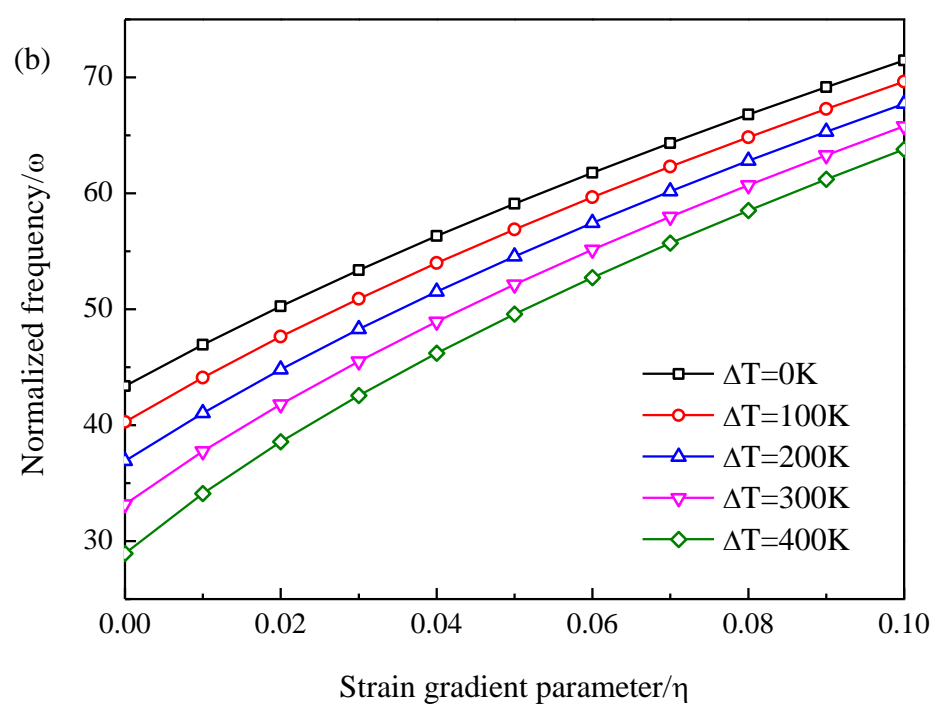

Figs.8 (a, b). Influences of variation of environmental temperature on normalized frequency for different nonlocal models (a) nonlocal stress gradient and (b) strain gradient parameter (FGX-CNTsRC, $V_{c n t}=0.17, V_{e}=5, V_{p}=1, A=0.2$ and $\alpha_{4}=1$ ).

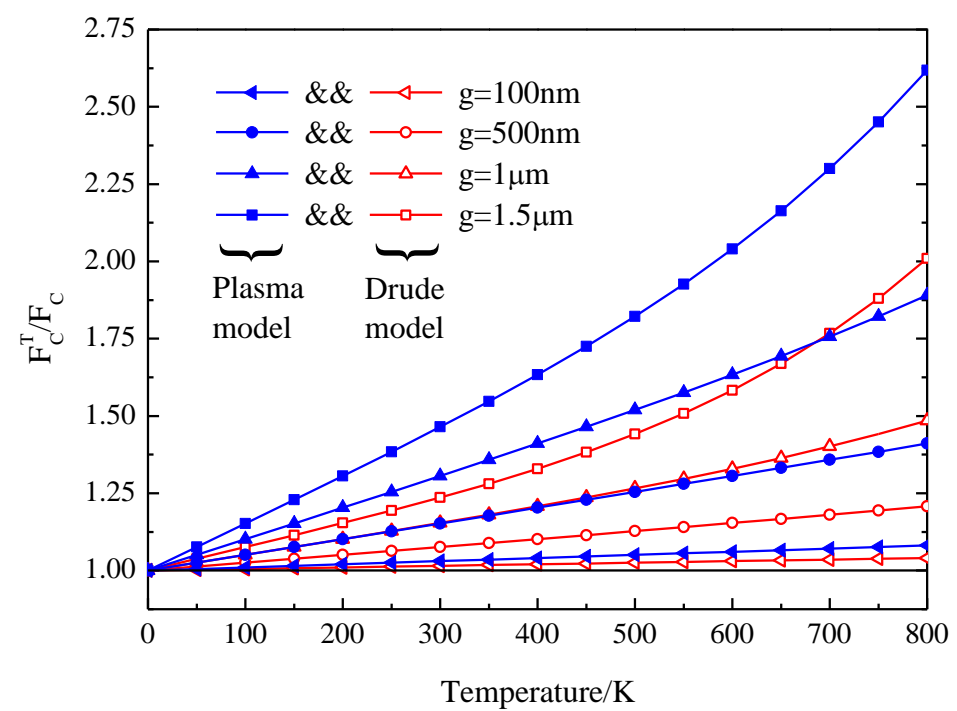

Fig. 9.Temperature-dependent thermal Casimir force based on Plasma model and Drude model under different initial gap 

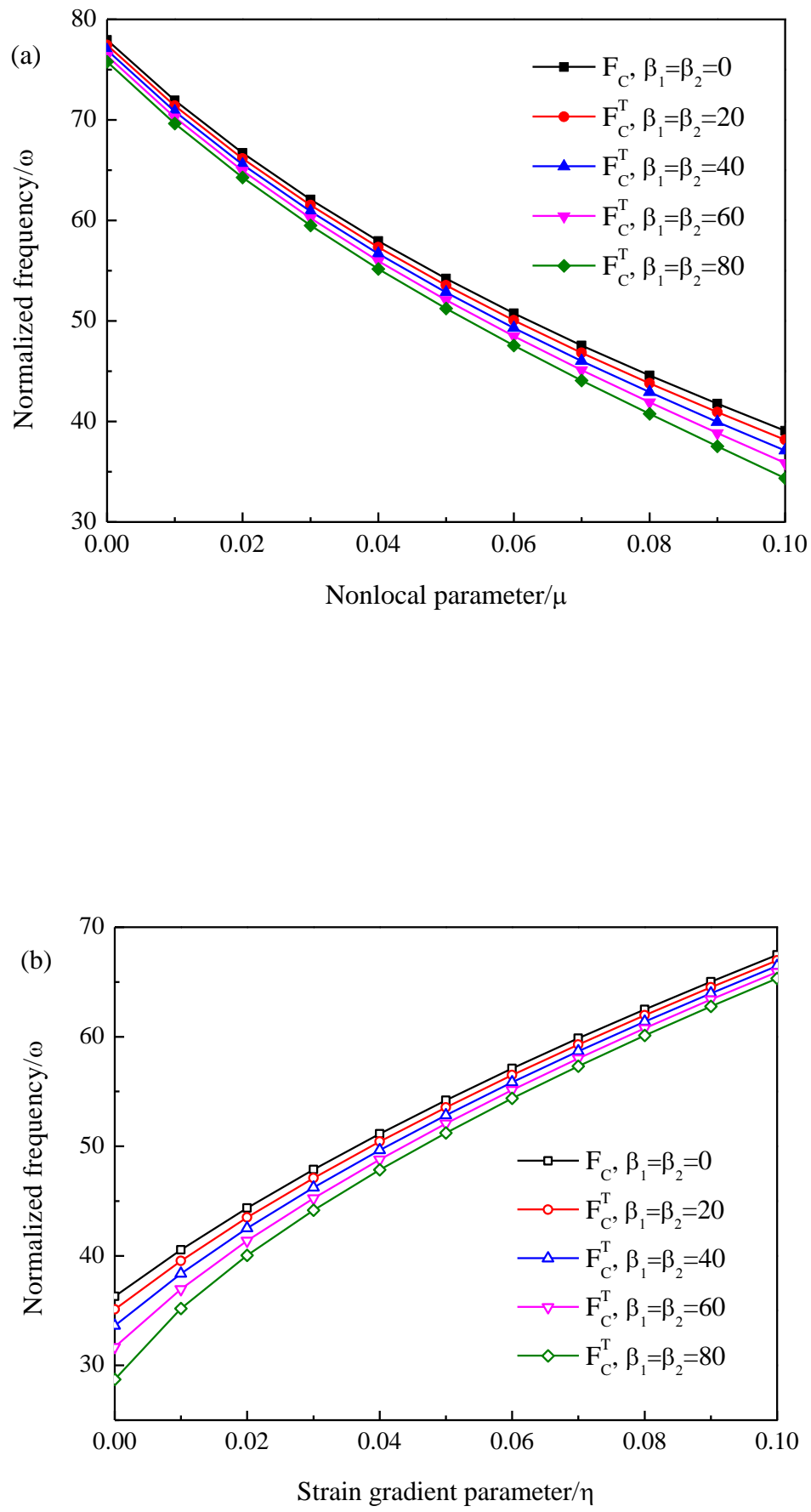

Figs. 10 (a, b).Comparison of quantum and thermal Casimir effect on normalized frequency for different nonlocal models (a) nonlocal stress gradient and (b) strain gradient parameter $\left(\right.$ FGX-CNTsRC, $V_{c n t}=0.17, V_{e}=5, V_{p}=1, A=0.2$ and $\left.\Delta T=200 \mathrm{~K}\right)$. 


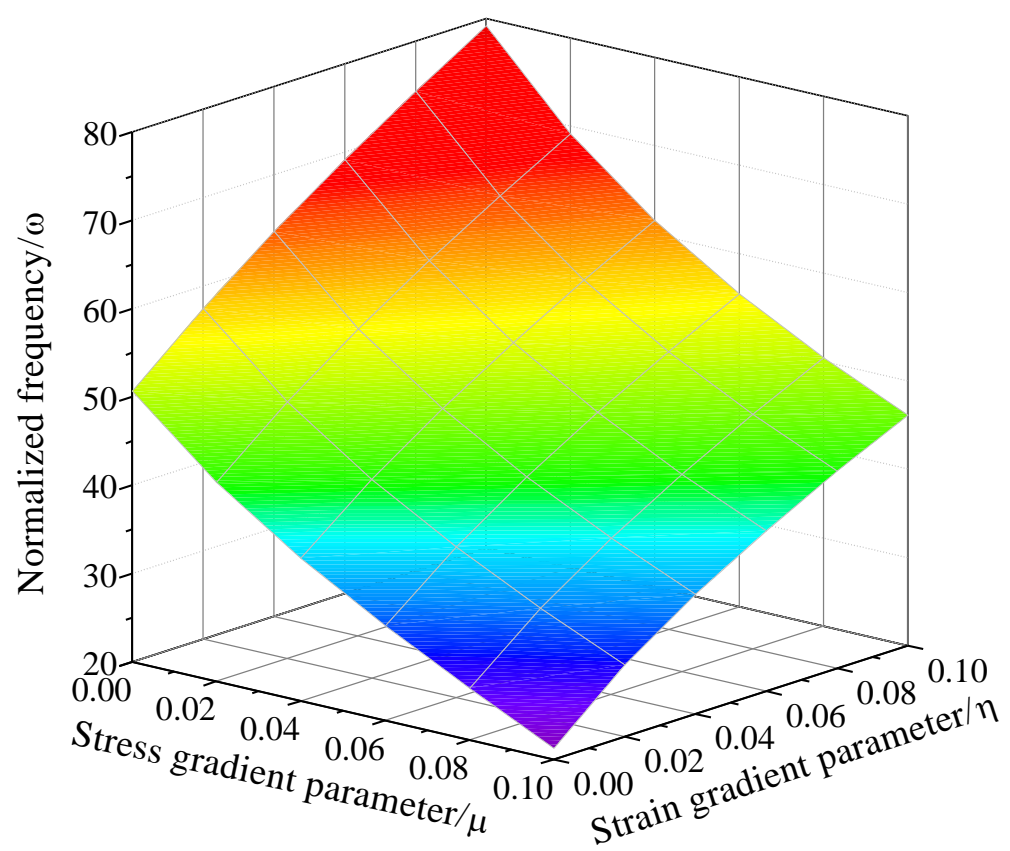

Fig. 11.Coupling effects of nonlocal stress and strain gradient parameters on normalized frequency of FGCNTs reinforced microbeam with piezoelectric layer.

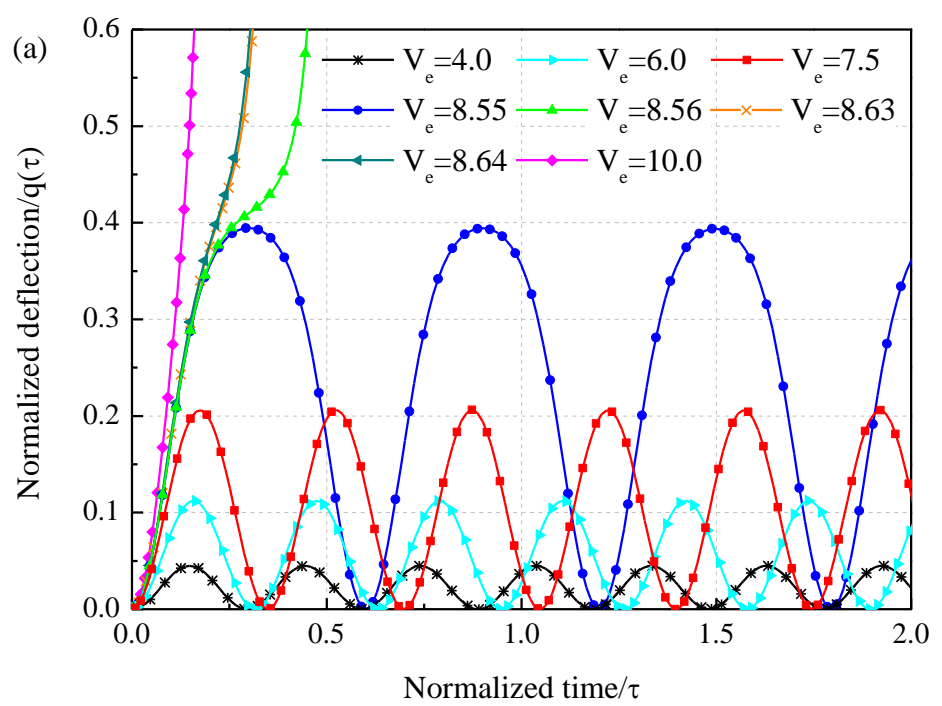




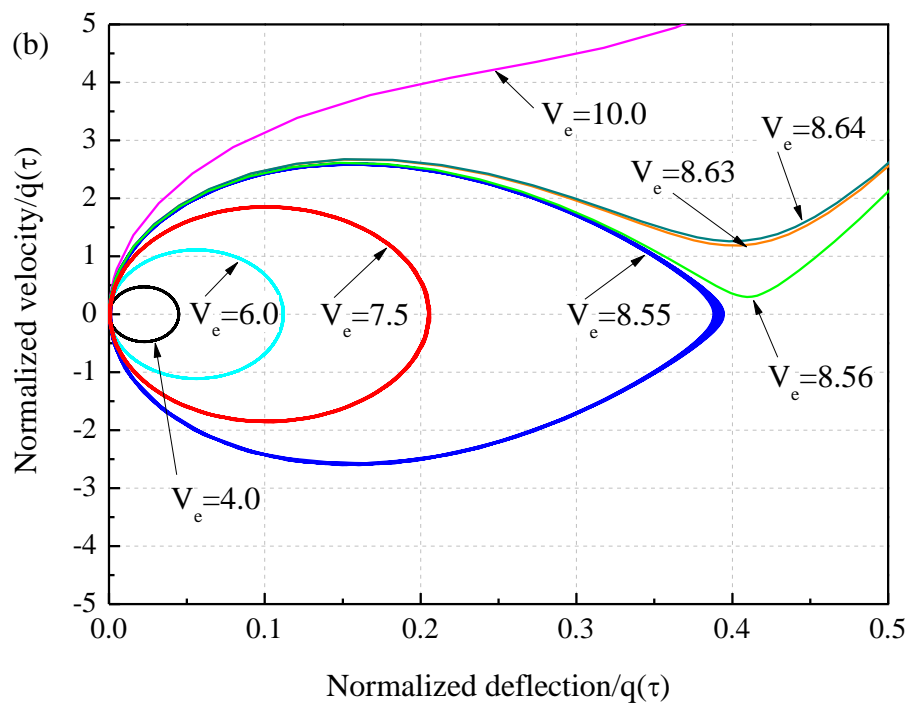

Figs. 12 (a, b). Dynamic behaviors of microbeam without damping effects near dynamic pull-in domain for (a) time story response and (b) phase plane near pull-in domain $\left(\mu=0.1, \eta=0.1, \hat{C}_{s d}=\hat{C}_{v d}=0\right)$.

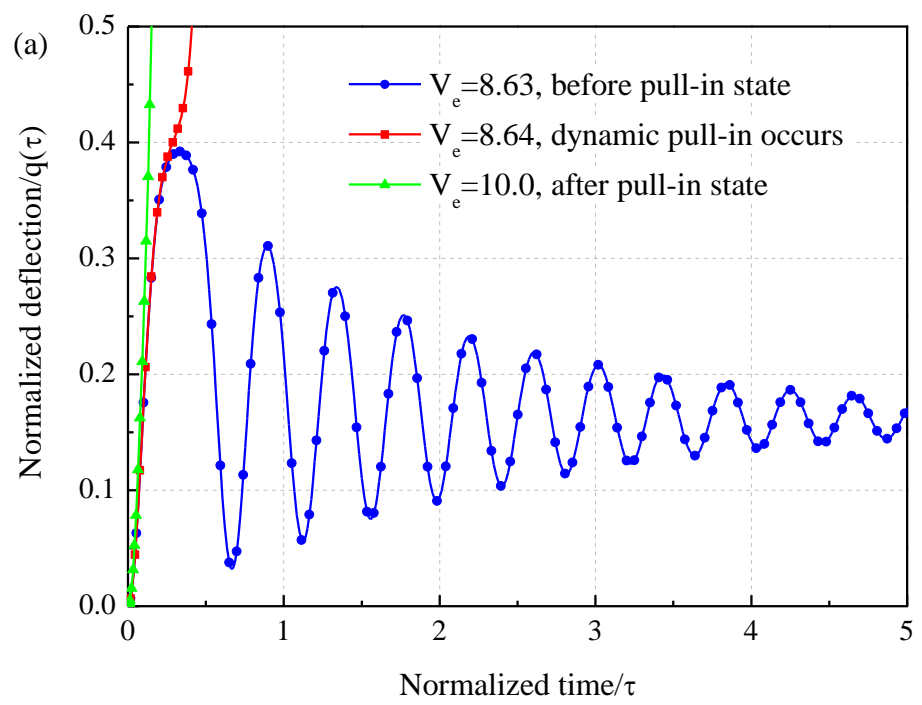




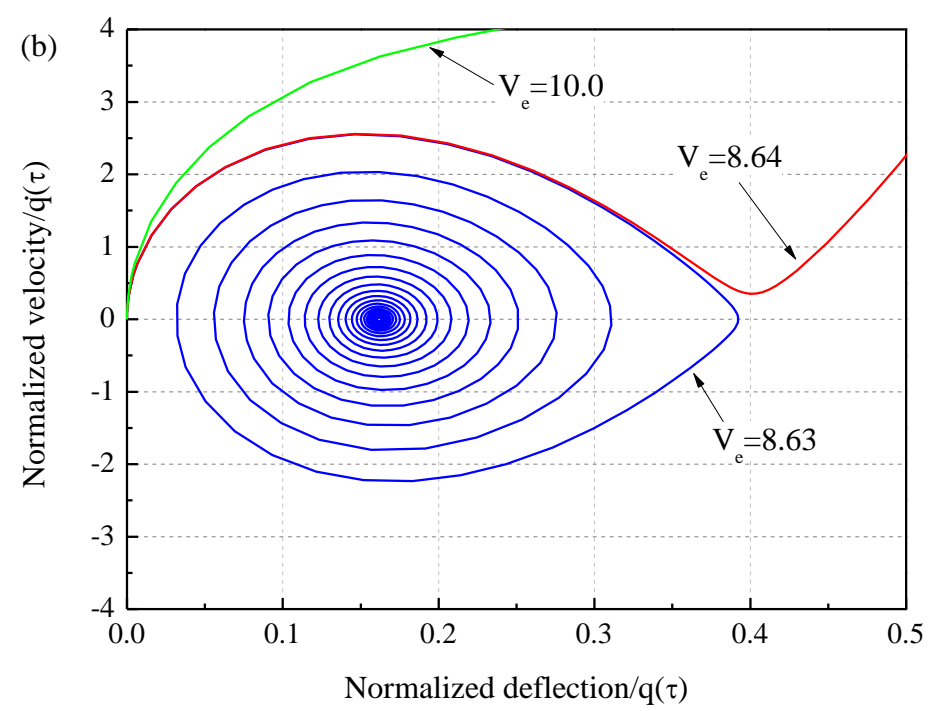

Figs.13 (a, b). Dynamic behaviors of microbeam with damping effects near dynamic pull-in domain for (a) time story response and (b) phase plane near pull-in domain

$$
\left(\mu=0.1, \eta=0.1, \hat{C}_{s d}=1 \text { and } \hat{C}_{v d}=1\right) .
$$

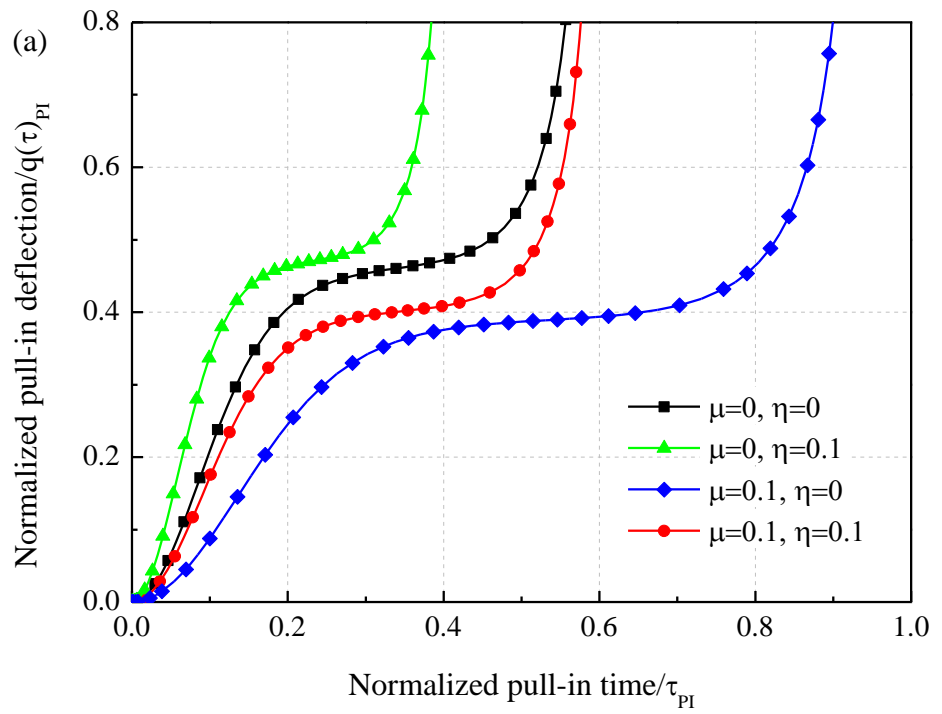




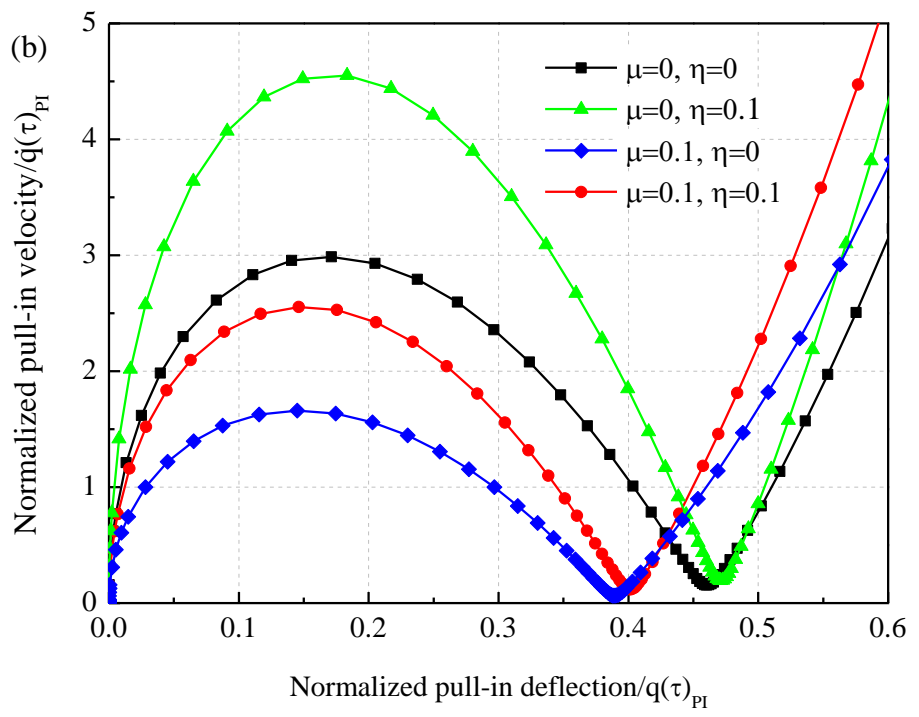

Figs. 14 (a, b).Dynamic behaviors of microbeam with damping effects at dynamic pull-in state for (a) time story response and (b) phase plane ( $\hat{C}_{s d}=1$ and $\left.\hat{C}_{v d}=1\right)$.

Table 1. Experimental parameters of dynamic pull-in for a clamped-clamped microbeam [44].

\begin{tabular}{llllll}
\hline \multirow{2}{*}{ Parameters } & Length & Width & Height & Initial gap & Young's modulus \\
& $(\mu \mathrm{m})$ & $(\mu \mathrm{m})$ & $(\mu \mathrm{m})$ & $(\mu \mathrm{m})$ & $(\mathrm{GPa})$ \\
Values & 610 & 40 & 2.12 & 2.07 & 164 \\
\hline
\end{tabular}

Table 2. Comparison of dynamic pull-in voltage obtained by different methods.

\begin{tabular}{llllll}
\hline $\begin{array}{l}\text { Residual stress } \\
(\mathrm{MPa})\end{array}$ & \multicolumn{5}{l}{ Dynamic pull-in voltage $(\mathrm{V})$} \\
\cline { 2 - 6 } & Experiment[44] & DIPIE algorithm[45] & EBM[46] & FEM[47] & Present \\
\hline$\sigma_{0}=-3.5$ & 8.10 & - & 8.027 & 7.980 & 7.985 \\
$\sigma_{0}=0$ & - & 10.752 & 10.974 & 11.074 & 11.108 \\
\hline
\end{tabular}


Table 3. Parameters of dynamic pull-in experiment with axial residual stress [48].

\begin{tabular}{llllll}
\hline \multirow{2}{*}{ Parameters } & Residual axial & Initial gap & Width & Height & Young's modulus \\
& load $(\mathrm{N})$ & $(\mu \mathrm{m})$ & $(\mu \mathrm{m})$ & $(\mu \mathrm{m})$ & $(\mathrm{GPa})$ \\
Values & 0.0009 & 1.18 & 100 & 1.5 & 166 \\
\hline
\end{tabular}

Table 4. Comparison of fundamental frequencies obtained by different methods.

\begin{tabular}{llllll}
\hline \multirow{2}{*}{$\begin{array}{l}\text { Beam length } \\
\text { ( } \mathrm{lm})\end{array}$} & \multicolumn{2}{l}{ Fundamental frequency (kHz) } & & \\
\cline { 2 - 5 } & Experiment[48] & Ritz method [49] & DQM[50] & HAM[51] & Present \\
\hline 210 & 322.05 & 324.21 & 324.70 & 324.78 & 324.34 \\
310 & 163.22 & 164.35 & 163.46 & 163.16 & 163.30 \\
410 & 102.17 & 103.80 & 103.70 & 103.42 & 103.72 \\
510 & 73.79 & 74.80 & 73.46 & 74.38 & 74.73 \\
\hline
\end{tabular}

Table 5. Temperature-dependent material property of CNTs and silicon $[52,53]$.

\begin{tabular}{lllll}
\hline Temperature $(\mathrm{K})$ & $E_{c n t}(\mathrm{TPa})$ & $\alpha_{c n t}\left(\times 10^{-6} / \mathrm{K}\right)$ & $E_{m}(\mathrm{MPa})$ & $\alpha_{m}\left(\times 10^{-6} / \mathrm{K}\right)$ \\
\hline 300 & 5.6466 & 3.4584 & 169.68 & 2.57 \\
400 & 5.5813 & 4.0919 & 167.69 & 3.14 \\
500 & 5.5308 & 4.5361 & 165.71 & 3.59 \\
600 & 5.4952 & 4.7438 & 163.72 & 3.88 \\
700 & 5.4744 & 4.6677 & 161.73 & 3.99 \\
\hline
\end{tabular}

Table 6. CNTs efficient parameter of scale-dependent property for different volume fraction $[30,31]$.

\begin{tabular}{lllll}
\hline$V_{c n t}^{*}$ & 0 & 0.12 & 0.17 & 0.28 \\
$\eta$ & - & 1.2833 & 1.3414 & 1.3238 \\
\hline
\end{tabular}




\section{Graphical abstract}

(a)
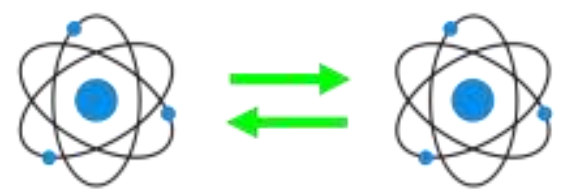

(b)
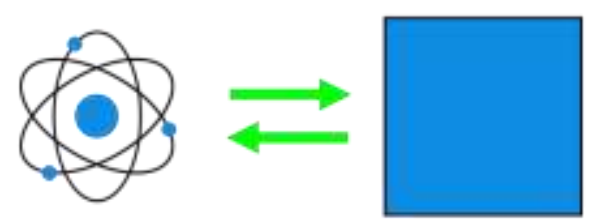

(c)
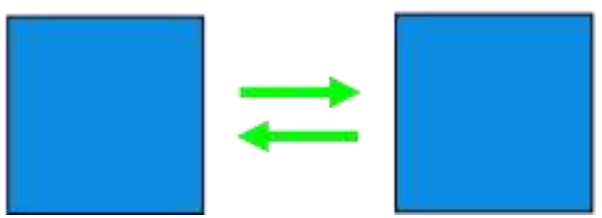

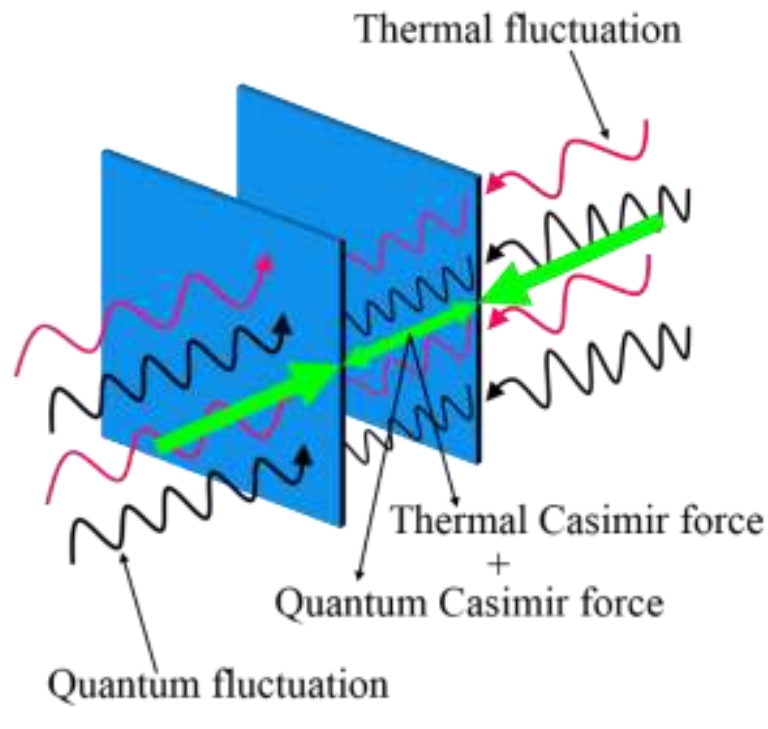

Fig. 1. Schematic of different micro-/nano-scale dispersion forces: (a) van der Waals force, (b) Casimir-Polder force, (c) Casimir force at zero temperature and (d) quantum and thermal fluctuation induced Casimir force at finite temperature environment.

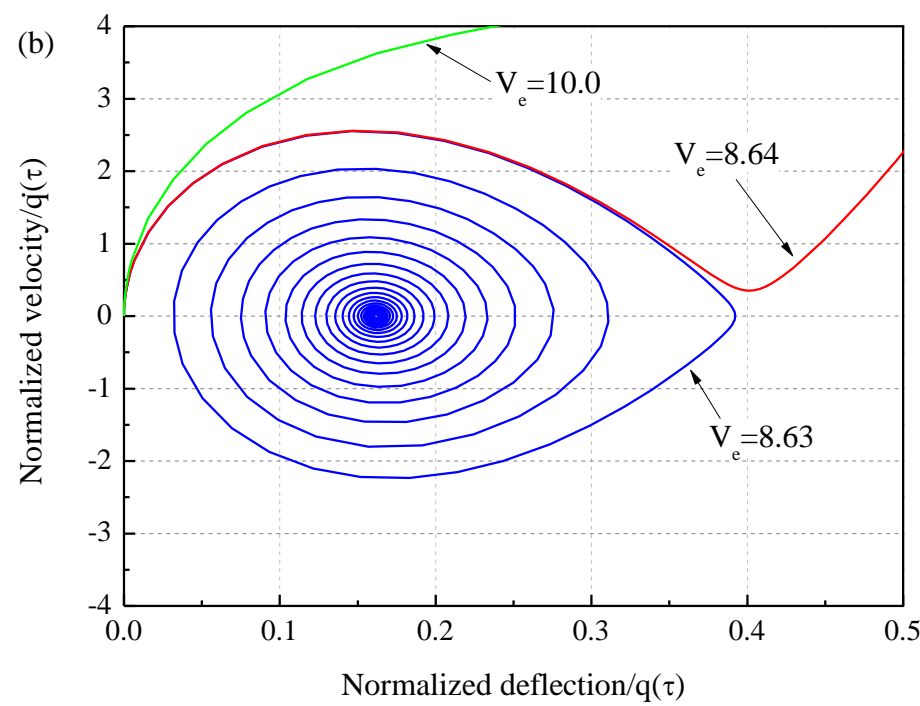

Fig.2 Phase plane near pull-in domain of microbeam with damping effects

$$
\left(\mu=0.1, \eta=0.1, \hat{C}_{s d}=1 \text { and } \hat{C}_{v d}=1\right) .
$$

